\title{
Kinetics and growth modes of quasi-2d silver branched electrodeposits produced in the presence of a supporting electrolyte
}

\author{
P.L. Schilardi, S.L. Marchiano, R.C. Salvarezza, A. Hernandez Creus ', A.J. Arvia * \\ Instituto de Investigaciones Fisicoquímicas Teóricas y Aplicadas (INIFTA), Universidad Nacional de La Plata, Sucursal 4, Casilla de Correo 16, 1900 La \\ Plata, Argentina
}

Received 2 September 1996; revised 17 December 1996

\begin{abstract}
Quasi-2d silver electrodeposits were grown electrochemically at . onstant potential from aqueous $\mathrm{Ag}^{+}$ion-containing solutions in the presence of a supporting electrolyte, at room temperature, using a three-electrode quasi-2d circular electrochemical cell. Open branching and dense radial branching patterns were distinguished on the centimetre scale, and growth mode transitions could be observed during the process. Branching patterns exhibited a mass fractal behaviour with a mass fracial dimension increasing from that expected for a DLA-like pattern to that of a dense branching pattern as either the cathodic overpotential $\left(\eta_{c}\right)$ or the $\mathrm{Ag}^{+}$ion concentration in the solution (c) was increased. The electrodeposition current increased with time exhibiting different regimes depending on whether an open branching or a dense radial branching growth mode prevailed. When the electrodeposition time exceeded a certain critical value, the radial growth rate of electrodeposits $\left(v_{\mathrm{r}}\right)$ approached a $v_{\mathrm{r}} \alpha \eta_{\mathrm{c}} c$ relationship. The experimental morphologies and growth kinetics were reproduced by Monte Carlo simulations of a growth model in which depositing particles follow a biased random walk. (C) 1997 Elsevier Science S.A.
\end{abstract}

Keywords: Silver; Electrodeposits; Kinetics; Growth: Supporting electrolyte

\section{Introduction}

The electrochemical formation of a solid phase on a solid substrate belongs to a group of common aggregation phenomena involving a rather complex mechanism which depends on the specific characteristics of the substrate, the environment and driving forces operating in the system. By adequately changing the experimental conditions different growth modes of the object can be produced [1-3].

When new phase formation takes place close to the thermodynamic equilibrium, layer by layer [4], three-dimensional (3d) island formation on two dimensional (2d) layers [5] and 3d island formation on the bare substrate [6] can be produced. These growth modes have been observed for a number of metal electrodeposits and, generally, lead to the formation of a rather compact phase which can usually be described as a euclidean object. Conversely,

\footnotetext{
* Corresponding author.

${ }^{1}$ Visiting Professor, Departamento de Química Física, Universidad de La Laguna, Tenerife, Spain.
}

under growth conditions far from equilibrium, irregular aggregates can be formed, depending on the operating conditions. The morphology of these deposits can frequently be described by the fractal geometry [1-3] as either self-similar or self-affine fractals.

In general, the formation of aggregates with self-affine fractal surfaces is enhanced when the phase growth kinetics is dominated by a surface reaction without significant surface relaxation $[7,8]$. On the other hand, self-similar fractals are promoted when the growth kinetics is controlled by transport phenomena. In this case, instabilities are favoured leading to the development of branched patterns.

Branched aggregate patterns can easily be followed through the formation of metal electrodeposits using quasi$2 \mathrm{~d}$ electrochemical cells involving either a concentric or a parallel electrode arrangement [9-15]. This type of experiment offers the possibility of varying the concentration of metal ions, the conductivity of the electrolyte solution, and the cell potential to develop different morphologies such as needle-like, dense radial branching (DBM) [13], dendritic (DM) $[11,12]$ and diffusion limited aggregation (DLA) 
[10-12,14,15]. DM and DLA patterns are both surface and volume fractals, DBM patterns are better described as surface fractals, and needle-like patterns are euclidean. Therefore, in $2 \mathrm{~d}$ cells the mass fractal dimension is $D_{M} \rightarrow 2$ for DBM patterns, $D_{\mathrm{M}} \approx 1.7$ for DLA and DM, and $D_{\mathrm{M}} \rightarrow 1$ for needle-like patterns [12-18]. According to the experimental conditions, transitions involving those morphologies at certain stages of growth have been observed [13-18]. These transitions have been studied extensively for $\mathrm{Cu}$ and $\mathrm{Zn}$ electrodeposition and in the electroformation of conducting polymers $[19,20]$. It has also been observed that, on the centimetre scale, aggregates change from the DLA-like form at low concentration and applied potential to the DBM at mid-applied potential range and to DM at high concentration and applied potentials [11,12,21,22]. The lack of reproducibility of metal growth patterns resulting from those runs made at the quasi-2d electrochemical cells has been attributed to the high sensitivity of these processes to impurities [18,23], a matter which was pointed out by Brady and Ball early on [24]. The influence of hydrodynamic conditions, electrocapillarity, recrystallization of the deposit, local dissolution of the aggregate [25], and pretreatment of the substrate [26] has also been discussed.

Despite the fact that the influence of concentration and applied voltage on the morphology of deposits has been adequately described [10-12], there are some electrochemical kinetic aspects of pattern evolution which are still under discussion. For instance, one should expect that DLA patterns are produced in the presence of a supporting electrolyte as in this case the motion of metal cations is assumed to be solely governed by diffusion [27].

This work describes the growth of quasi- $2 \mathrm{~d} \mathrm{Ag}$ electrodeposits on a Pt cathode from aqueous $\mathrm{Ag}^{+}$ion-containing solutions in the presence of a supporting electrolyte under potentiostatic control including ohmic drop corrections. Results on the centimetre scale show a change in the growth patterns from a DM or a DLA-like to a DBM pattern as either the applied potential or the $\mathrm{Ag}^{+}$ion concentration in the solution is increased followed by another transition from a DMB to a DM pattern as the size of the deposit approaches the size of the cell. It is further shown that when the electrodeposition time exceeds a certain critical value, the front propagation rate, taken as the displacement velocity of the largest branch of the deposit, increases almost linearly eithei with the $\mathrm{Ag}^{+}$ion concentration at a constant cathodic overpotential, or with the cathodic overpotential at a constant $\mathrm{Ag}^{+}$ion concentration.

The morphology patterns and the main kinetic features related to the formation of the $\mathrm{Ag}$ branching patterns produced in the presence of a supporting electrolyte can be qualitatively approached from rather simple Monte Carlo simulations based upon a biased random walk for depositing particles through a composed attachment probability. This statistical approach is consistent with an electrochem-

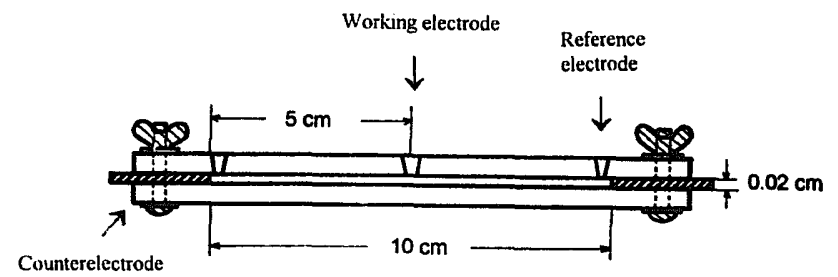

Fig. 1. Scheme of the quasi-2d cylindrical electrochemical cell.

ical process driven by an electrochemical potential gradient.

\section{Experimental}

The experimental set-up consisted of a cylindrical electrochemical cell made of two pitiallel glass plates separated by a $0.02 \mathrm{~cm}$ height apprraching a circular $2 \mathrm{~d}$ cell geometry (Fig. 1). An axially placed $\mathrm{Pt}$ wire $(99.99 \%$ purity and $0.035 \mathrm{~cm}$ radius, $R_{0}$ ) was used as cathode, and a $\mathrm{Ag}$ ring $(0.02 \mathrm{~cm}$ thickness and $5.0 \mathrm{~cm}$ inner radius) placed around the cathode was employed as anode. $\mathrm{A} \mathrm{Ag}$ wire
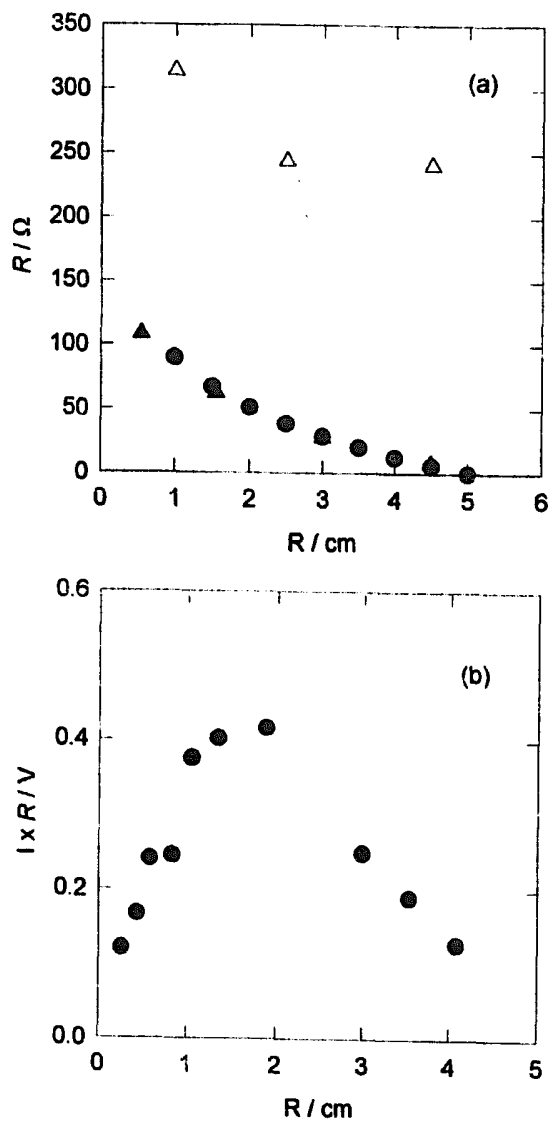

Fig. 2. (a) Silver anode-to-silver cathode cell resistance $(R)$ vs. the radius $(R)$ of the quasi-circular cathode: 0 , calculated data; $\Delta$, experimental data from quasi-circular electrodes; $\Delta$, experimental data from $\mathrm{Ag}$ branched deposits. (b) Silver anode-to-silver cathore ohmic drop vs. the radius of the quasi-circular cathode for $\eta_{\mathrm{c}}=0.760 \mathrm{~V}$. Electrolyte solution: aqueous $0.024 \mathrm{M} \mathrm{Ag}_{2} \mathrm{SO}_{4}+0.01 \mathrm{M} \mathrm{H}_{2} \mathrm{SO}_{4}+0.5 \mathrm{M} \mathrm{Na}_{2} \mathrm{SO}_{4}$. 
immersed in the same working solution and connected to the cell through a capillary tip was used as the reference electrode.

The cell design favoured a homogeneous primary current distribution. The cell was placed in an air table to avoid the interference of mechanical vibrations in mass transport processes on the solution side, and kept at $298 \mathrm{~K}$.

Aqueous $c \mathrm{M} \mathrm{Ag} \mathrm{SO}_{4}+0.5 \mathrm{M} \mathrm{Na} \mathrm{Na}_{2}+10^{-2} \mathrm{M}$ $\mathrm{H}_{2} \mathrm{SO}_{4}$ solutions in the range $1 \times 10^{-3} \leq c \leq 2.4 \times 10^{-2}$ were employed. Solutions were prepared from Milli- $\mathrm{Q}^{*}$ water and a.r. chemicals and deaerated, for at least $2 \mathrm{~h}$, by bubbling purified $\mathrm{N}_{2}$ gas before the electrochemical cell was filled.

$\mathrm{Ag}$ aggregates were formed at a constant cathodic overpotential $\left(\eta_{c}\right)$ covering the range $0.118 \mathrm{~V} \leq \eta_{c} \leq 0.760 \mathrm{~V}$. The cathodic overpotential was defined as $\eta_{\mathrm{c}}=E_{\mathrm{eq}}-E$, where $E_{\text {eq }}$ was the reversible potential of the $\operatorname{Ag}_{\mid} \operatorname{Ag}^{+}(c, \mathrm{aq})$ electrode, and $E$ was the applied potential. These measurements were performed using the supporting electrolyte-containing solution changing adequately both $c$ and $\eta_{c}$ separately. For the above experimental conditions no interference due to the hydrogen evolution reaction could be observed.
Electrochemical runs were made using an LYP M6 potentiostat, an LYP CD02 digital coulometer, and a Hewlett-Packard $X-Y$ recorder.

Current transients ( $I$ vs. $t$ plots) and charge transients ( $Q$ vs. $t$ plots) were recorded from $t=0$, the time at which $E$ was switched on, to certain values of time $i$, which were selected according to the duration of each experiment. Simultaneously, photographs of the growing object were taken søquentially.

\section{Results}

\subsection{Cell design and cathode-to-anode potential drop}

The small thickness cell design required for this work implied a significant potential drop between the cathode and the anode as well as between the cathode and the reference electrode. The accurate evaluation of the potential drop was limited by the irregularity of the growing deposit which depends on the applied potential, solution composition and electrolysis time, as discussed in the following sections.

(a)

$1 \mathrm{~cm}$

(b)

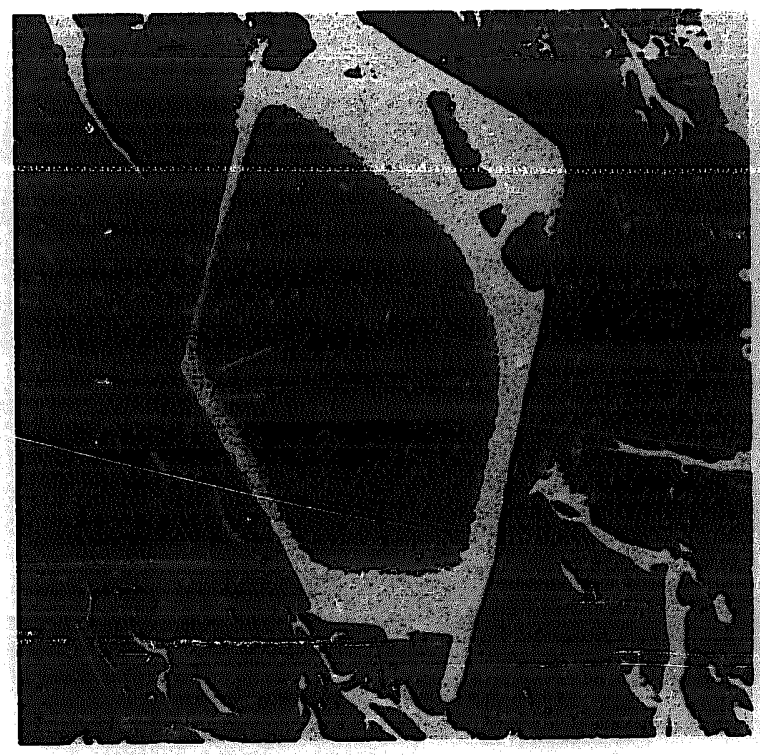

$4.85 \mu \mathrm{m}$ (c)

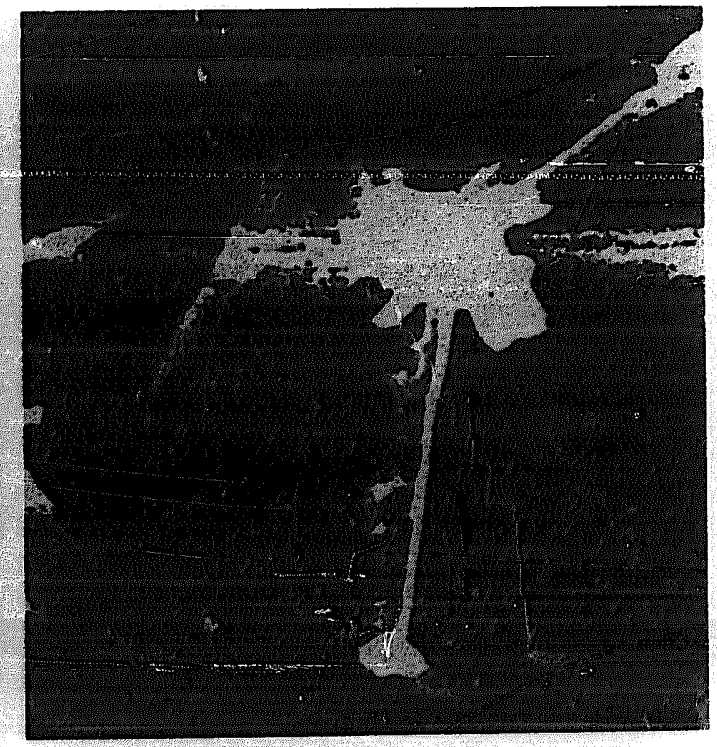

$4.15 \mu \mathrm{m}$
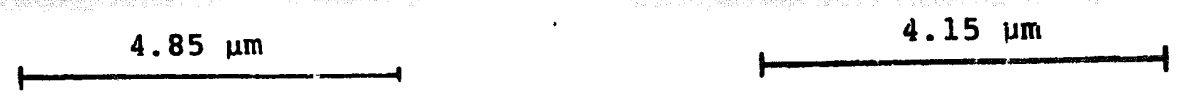

Fig. 3. (a) Initial stages of silver electrodeposit growth (compact object) and initiation of the irregular profile. The first dot represents the substrate profile. (b,c) SEM micrographs showing the triggering of dendrites from the border of compact silver crystallites. 
To estimate the ohmic drop a dummy cell with $\mathrm{Ag}$ disc cathodes of different diameters comprised between 0.5 and $4 \mathrm{~cm}$ to simulate the average size of electrodeposits was used. Besides, the resistance between Ag electrodeposits at different stages of growth and the reference electrode tip was also determined. For this purpose, an aqueous $0.024 \mathrm{M}$ $\mathrm{Ag}_{2} \mathrm{SO}_{4}+0.05 \mathrm{M} \mathrm{Na}_{2} \mathrm{SO}_{4}+0.01 \mathrm{M} \mathrm{H}_{2} \mathrm{SO}_{4}$ solution was employed, its specific conductivity being $\kappa=5.7 \times$ $10^{-2} \Omega^{-1} \mathrm{~cm}^{-1}$ at $298 \mathrm{~K}$. For both types of experiments the value of $R$, the cathode-to-anode resistance, decreased as $\mathbf{R}$, the radius of the cathode, was increased according to Ohm's law for a cylindrical geometry. Results obtained with both the dummy and the working cells are shown in Fig. 2(a).

The ohmic drop ( $I R)$ was calculated taking values of $R$ from Fig. 2(a), and current values from the corresponding current transient read for Ag electrodeposits of comparable average radius, assuming a similar resistivity for both the Ag electrodeposit and the $\mathrm{Ag}$ disc cathode. This assumption is justified by the fact that the in situ experimental resistivity of our DBM Ag patterns resulted in $10 \Omega \mathrm{cm}$, a figure which is sinilar to that previously reported for $\mathrm{Cu}$ DMB patterns [22]. Furthermore, resistance measurements carried out on mechanically stable arms of $\mathrm{Ag}$ branched deposits resulted in a resistivity close to $0.2 \Omega \mathrm{cm}$. In all cases the $I R$ drop correction was applied to $\mathrm{Ag}$ patterns with values of $R$ smaller than $2 \mathrm{~cm}$. This means that the contribution of the $\mathrm{Ag}$ electrodeposit to the electrical resistance is much smaller than that of the electrolyte solution. This procedure was basically applied to those electrodeposits grown concentrically, althcugh it could be extended to those branching objects growing symmietrically around the cathode.

The $I R$ vs. $R$ plot reaches a maximum at a certain value of R (Fig. 2(b)) and shows that for the solution compositions used in this work, the ohmic drop correction makes the effective potential acting at the electrochemical interface considerably different from the cathode reference electrode potential, depending on the electrodeposit size.

\subsection{The growth modes of $\mathrm{Ag}$ electrodeposits in a support- ing electrolyte-containing solution}

The growth of Ag aggregates involves distinguishable growth modes which can be observed at different scales and stages of the process. The early stages of growth (Fig. 3(a)) show an initially compact deposit following the Pt cathode geometry and extending up to the radius of the growing object, which for the case of Fig. 3(a) is approximately twice the radius of the cathode. Afterwards, as can already be seen on the scale of Fig. 3(a), the object commences to develop an irregular shape. At this stage a SEM micrograph of the deposit (Fig. 3(b), (c)) shows that it consists of irregular crystallites in which the triggering of a filament-like branching takes place, in agreement with experiments previously described for the growth of $3 \mathrm{~d} \mathrm{Ag}$ electrodeposits [28]. The transition from the initially compact crystallites to the branching growth mode (first growth (a)
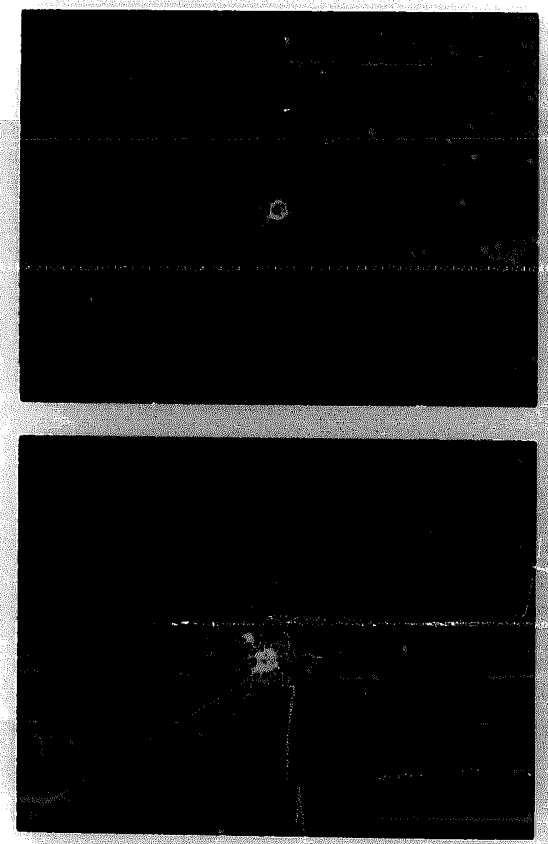

(d) (b) $\overline{1 \mathrm{~cm}}$
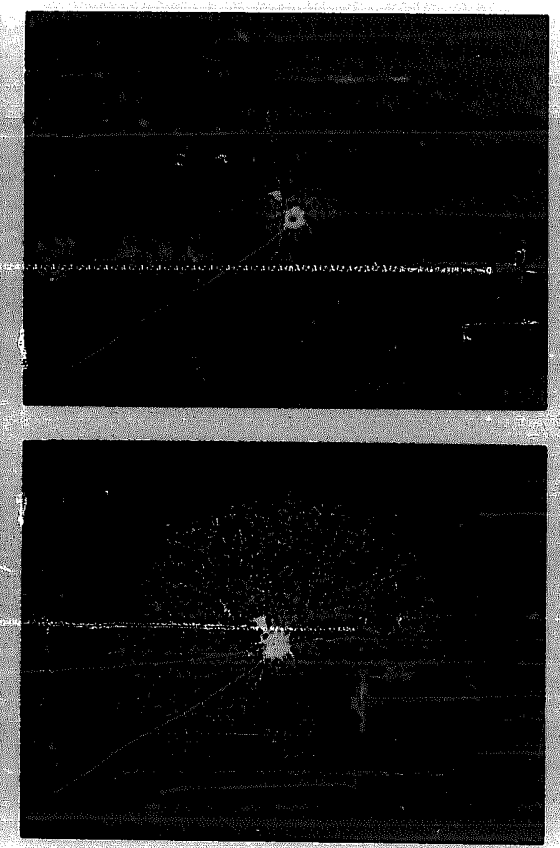

(e) (c)

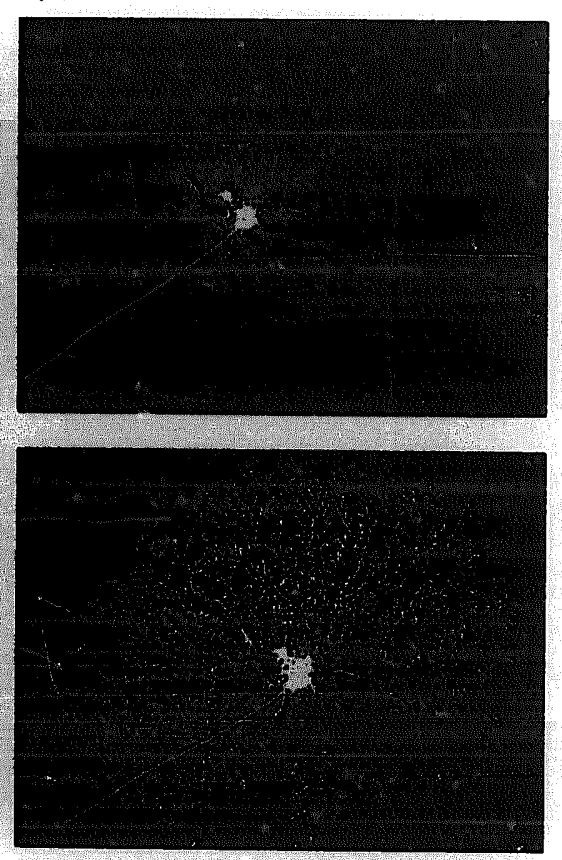

(f) Fig. 4. Photograpis showing the $\epsilon$ volution of a silver electrodeposit grown at $\eta_{\mathrm{c}}=0.318 \mathrm{~V}$ in aqueous $0.024 \mathrm{M} \mathrm{Ag}_{2} \mathrm{SO}_{4}+0.01 \mathrm{M} \mathrm{H} \mathrm{SO}_{4}+0.5 \mathrm{M}$
$\mathrm{Na}_{2} \mathrm{SO}_{4}$. (a) $t=210 \mathrm{~s}$; (b) $t=54 \mathrm{~S}$; (c) $t=1020 \mathrm{~s}$; (d) $t=1620 \mathrm{~s}$; (e) $t=3420 \mathrm{~s}$; (f) $t=5220 \mathrm{~s}$. 
(a)

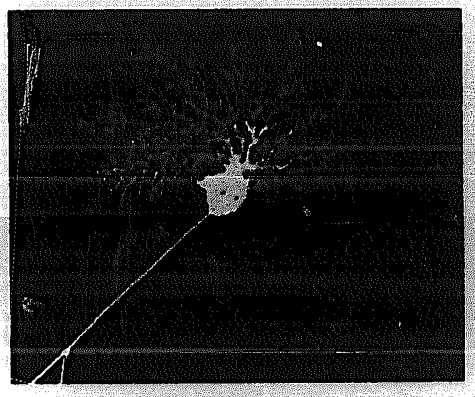

$1 \mathrm{~cm}$

(c)

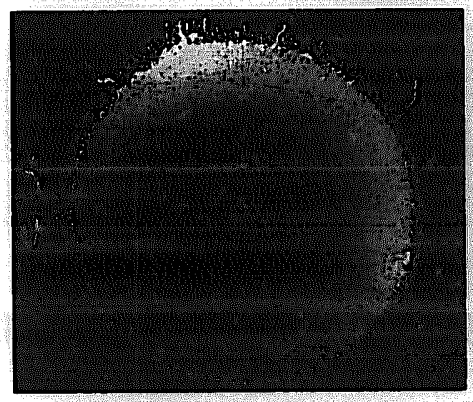

$1 \mathrm{~cm}$ (b)

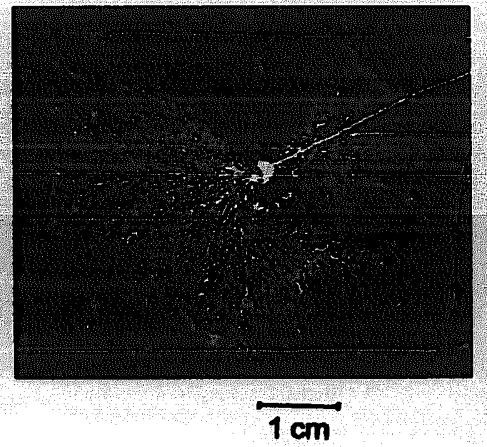

(d)

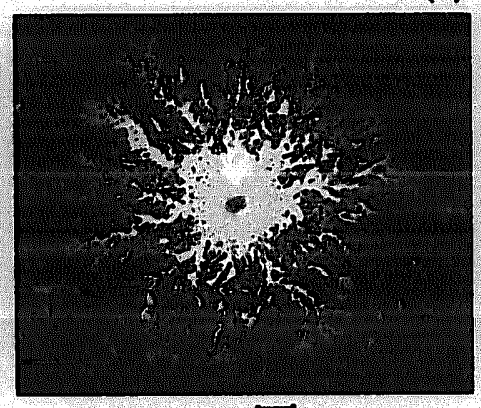

$\stackrel{\overrightarrow{\mathrm{cm}}}{\mathrm{c}}$ (e)

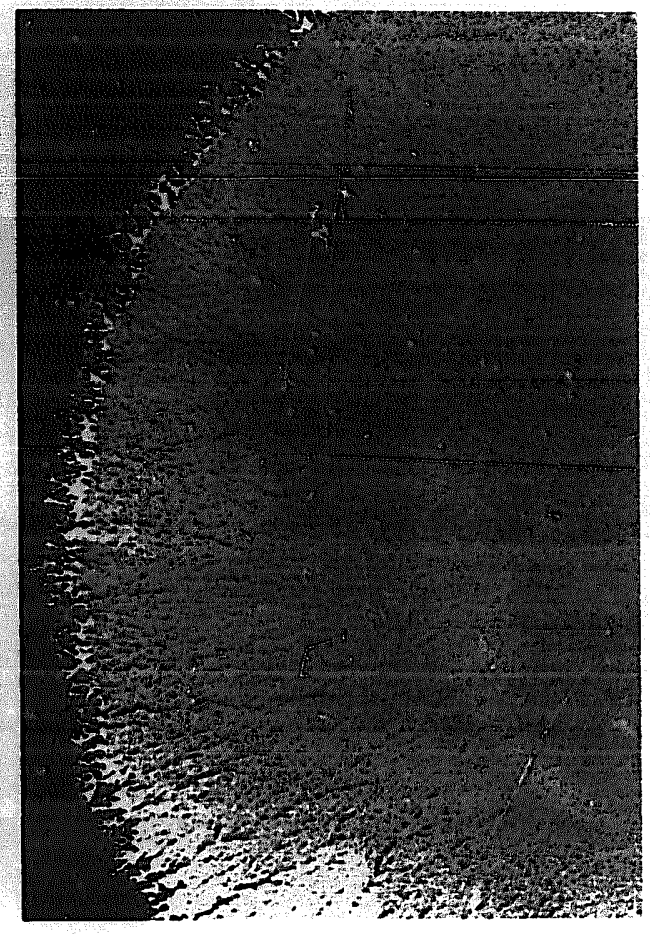

Fig. 5. Photographs showing the influence of $c$ on the shape of $\mathrm{Ag}$ electrodeposits grown at $\eta_{\mathrm{c}}=0.660 \mathrm{~V}$, at different charges $Q$. (a) $c=0.002 \mathrm{M}$, $Q=0.165 \mathrm{C}$; (b) $c=0.005 \mathrm{M}, Q=1.381 \mathrm{C}$; (c) $c=0.014 \mathrm{M}, Q=1.03 \mathrm{C}$; (d) $c=0.024 \mathrm{M}, Q=1.43 \mathrm{C}$; (e) detail of the dense radial to open radial transition region from (c). Electrolyte solution: aqueous $c \mathrm{M} \mathrm{Ag}_{2} \mathrm{SO}_{4}+0.01 \mathrm{M} \mathrm{H}_{2} \mathrm{SO}_{4}+0.5 \mathrm{M} \mathrm{Na}_{2} \mathrm{SO}_{4}$.

(a)

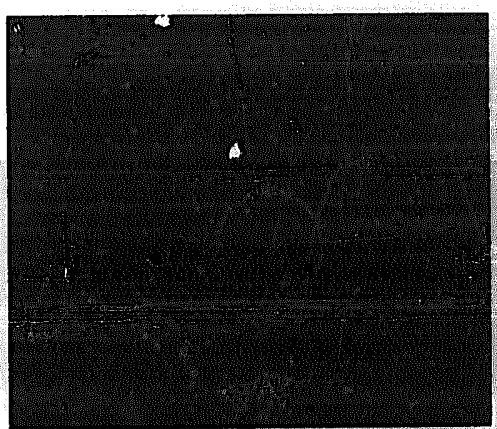

(d)

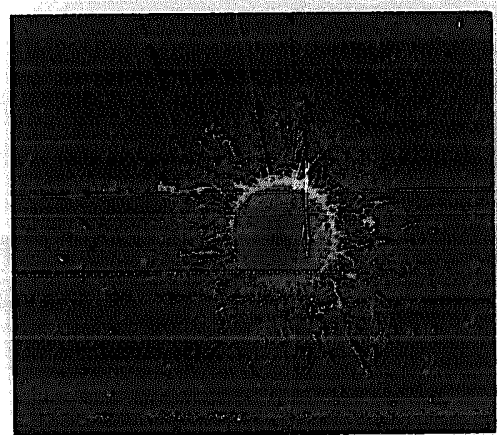

$1 \mathrm{~cm}$

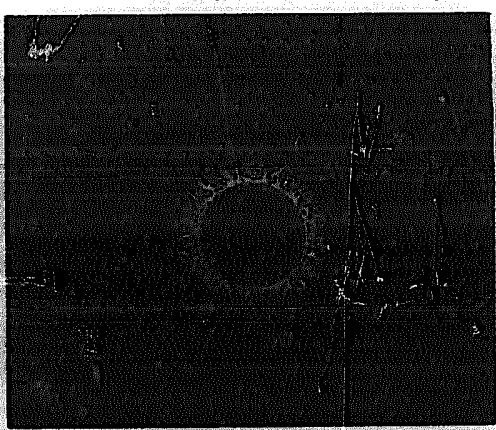

(e)

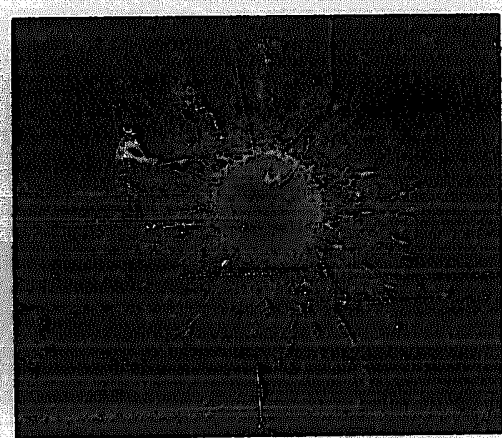

(c)

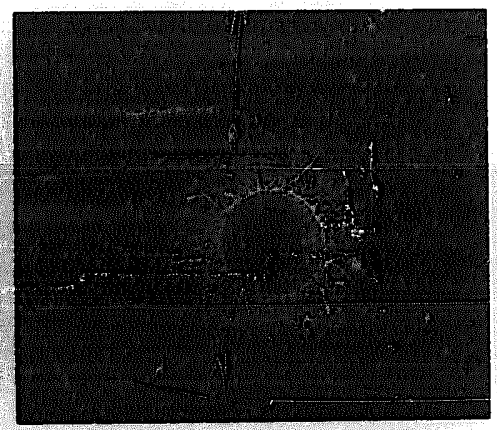

(f)

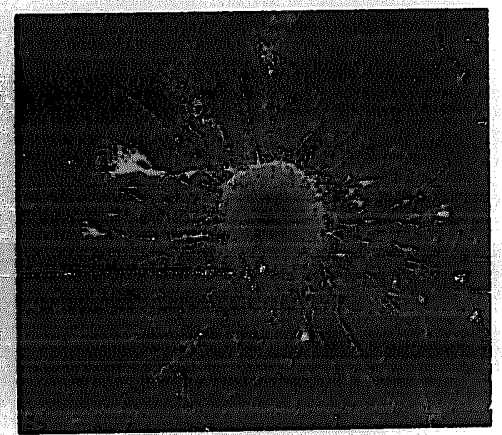

Fig. 6. Photographs showing the growth transitions in the $\mathrm{Ag}$ electrodeposits produced at $\eta_{\mathrm{c}}=0.760 \mathrm{~V}$. Electrolyte solution: aqueous $0.024 \mathrm{M}$ $\mathrm{Ag}_{2} \mathrm{SO}_{4}+0.01 \mathrm{M} \mathrm{H}_{2} \mathrm{SO}_{4}+0.5 \mathrm{M} \mathrm{Na}_{2} \mathrm{SO}_{4}$. 
mode transition) operates at the level of those tiny crystals in which branch triggering appears as fine tips emerging from borders and corners of crystal terraces. This first growth mode transition of $\mathrm{Ag}$ electrodeposits deserves investigation on the micro- and nanometre scale, as has been made for the case of $\mathrm{Au}$ electrodeposits on the C(0001) basal plane using STM imaging [29]. This transition is beyond the experimental possibilities and scope of this work.

Following the first growth mode transition an irregularly radial $\mathrm{Ag}$ electrodeposit is produced, as can be seen in Fig. 4(a)-(f), for those runs made for $c=0.024$ and $\eta_{i}=0.318 \mathrm{~V}$. Transitions in the growth mode of these deposits as described above can always be observed, irrespective of $c$ and $\eta_{c}$. Accordingly, the kinetics and growth mode of $\mathrm{Ag}$ electrodeposits were followed as a function of $c$ and $\eta_{c}$ by measuring the time dependence of the current $(I)$, the charge passed $(Q)$ and the radius $(\mathrm{R})$ which is proportional to the radius of gyration of the deposit.

The fractal dimension $\left(D_{\mathrm{M}}\right)$ of electrodeposits was also determined from the $\log Q$ vs. $\log \mathrm{R}$ plots. However, for electrodeposits which exhibit complex patterns with differ- ent morphologies, presumably a multifractal approach would be more appropriate [30]. Thus, in this work the value of $D_{M}$ is taken only as an indication of the transition from a dense branched to an open branched growth mode as either $\eta_{c}$ or $c$ is changed.

\subsection{The influevce of silver ion concentration on the growth mode of Ag elestrodeposits}

The influence of $c$ on the growth mode of $\mathrm{Ag}$ electrodeposits proluced at $\eta_{\mathrm{c}}=0.660 \mathrm{~V}$ was investigated in the range $1 \times 10^{-3} \leq c \leq 2.4 \times 10^{-2}$.

\subsubsection{Transitions in the growth mode}

$\mathrm{Ag}$ deposits resulting from $c \leq 5 \times 10^{-3}$ at $\eta_{\mathrm{c}}=$ $0.660 \mathrm{~V}$ (Fig. 5(a), (b)) appear as anisotropic open patterns in which the thickness of the inner compact deposit is almost negligible compared to the size of the object. Furthermore, the outer open radial branched structure for $c=0.002$ shows a lower branching density than that for $c=0.005$. Conversely, for $c \geq 10^{-2}$ an axially symmetric deposit is formed (Fig. 5(c)) which exhibits an inner dense
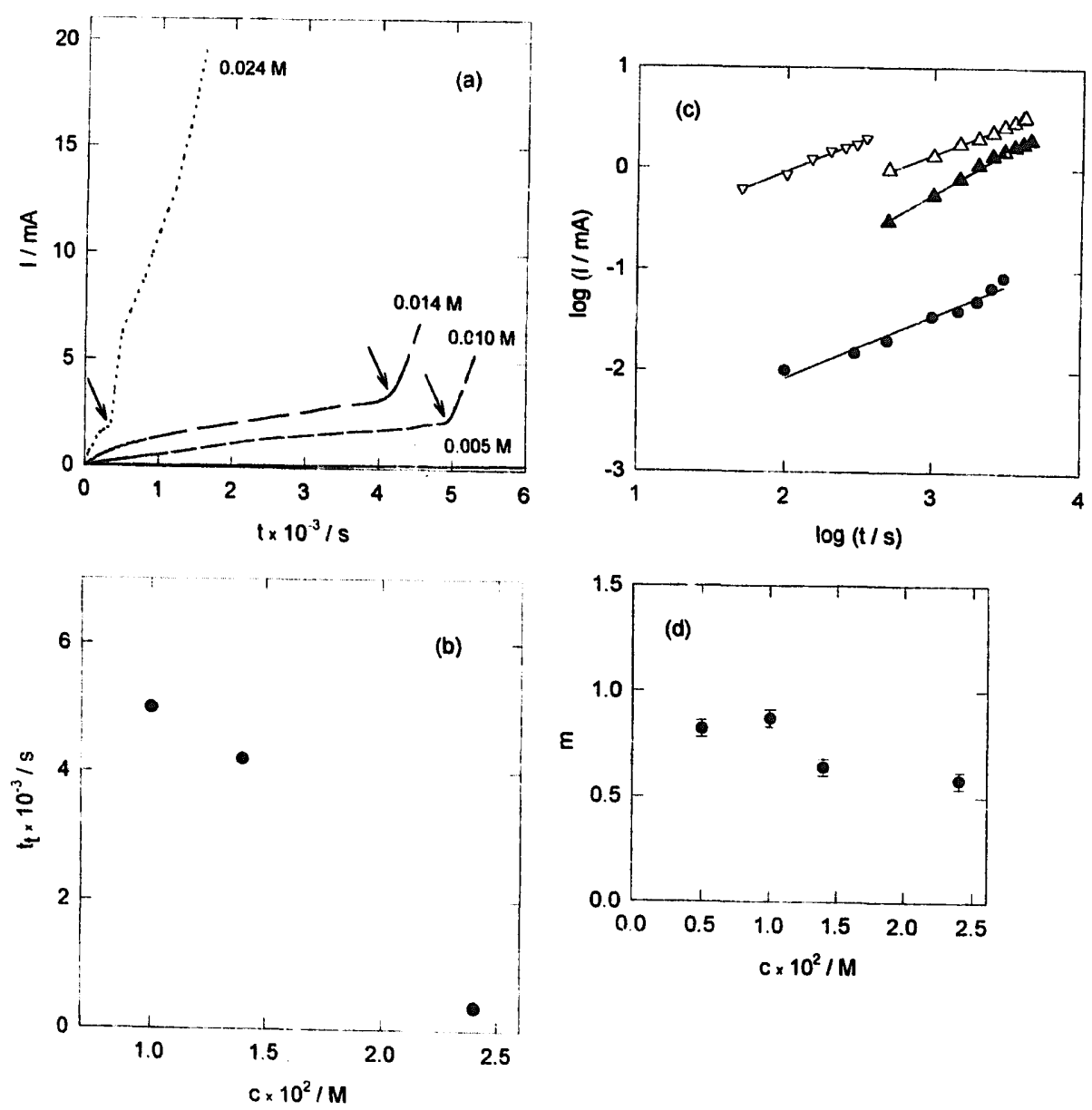

Fig. 7. (a) Current transients resulting from silver electrodeposition at $\eta_{\mathrm{c}}=0.660 \mathrm{~V}$ and different values of $c$, as indicated in the figure. Transition times ( $\left.t_{1}\right)$ are indicated by arrows. (b) Dependence of $t_{1}$ on $c$. Electrolyte solution: aquecus $c \mathrm{M} \mathrm{Ag}_{2} \mathrm{SO}_{4}+0.01 \mathrm{M} \mathrm{H}_{2} \mathrm{SO}_{4}+0.5 \mathrm{Mi} \mathrm{iaa}_{2} \mathrm{SO}_{4}$. (c) $\mathrm{Lag} i$ vs. $\log t$ plots at $\eta_{c}=0.660 \mathrm{~V}$ and different values of $c$ : $(\odot) 0.005,(\Delta) 0.01,(\Delta) 0.014,(\nabla) 0.024$. (d) $m$ vs. $c$ plot. Electrolyte solution: aqueous $c \mathrm{M}$
$\mathrm{Ag}_{2} \mathrm{SO}_{4}+0.01 \mathrm{M} \mathrm{H}_{2} \mathrm{SO}_{4}+0.5 \mathrm{M} \mathrm{Na}_{2} \mathrm{SO}_{4}$. 

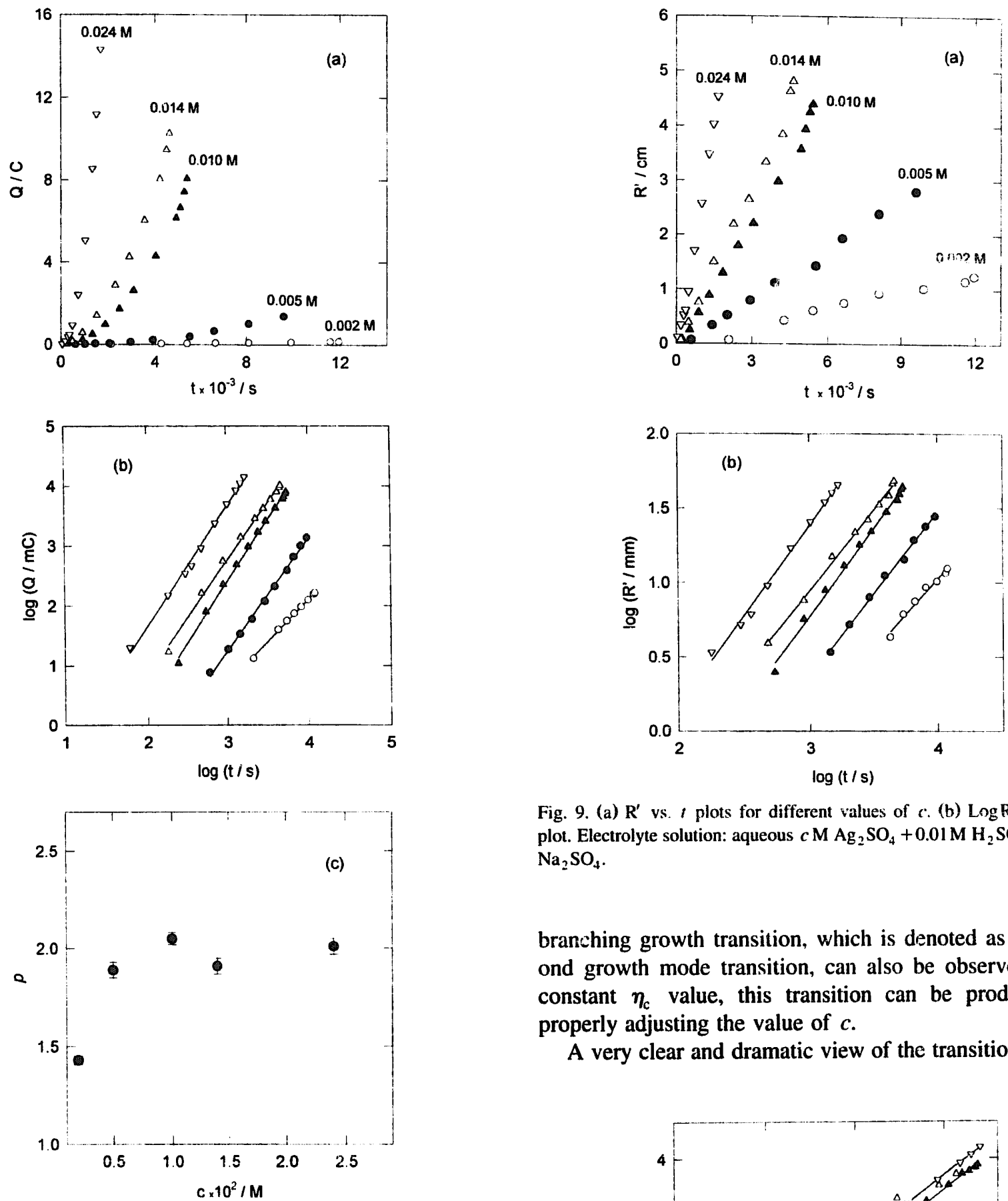

Fig. 8. (a) $Q$ vs. $t$ plots resulting from those runs made at $\eta_{\mathrm{c}}=0.660 \mathrm{~V}$ and different values of $c$, as indicated in the figure. (b) $\log Q$ vs. $\log t$ plots: (O) $c=0.002,(O) c=0.005,(\Delta) c=0.01,(\Delta) c=0.014,(\ulcorner)$ $c=0.024$. (c) $p$ vs. $c$ plot. Electrolyte solution: aqueous $c \mathrm{M} \mathrm{Ag}_{2} \mathrm{SO}_{4}+$ $0.01 \mathrm{M} \mathrm{H}_{2} \mathrm{SO}_{4}+0.5 \mathrm{M} \mathrm{Na}_{2} \mathrm{SO}_{4}$.

radial region extending up to a certain radius. Afterwards, an outer layer with a low arm density begins to be observed. In contrast to the open branched region, as $c$ is still further increased (Fig. 5(d)), the thickness of the dense branched region decreases. This region consists of arms thicker than those found for $c \leq 5 \times 10^{-3}$. Those patterns shown in Fig. 5(c), (e) reveal that a dense-to-open radial

branching growth transition, which is denoted as the second growth mode transition, can also be observed. At a constant $\eta_{c}$ value, this transition can be produced by properly adjusting the value of $c$.

A very clear and dramatic view of the transition from a

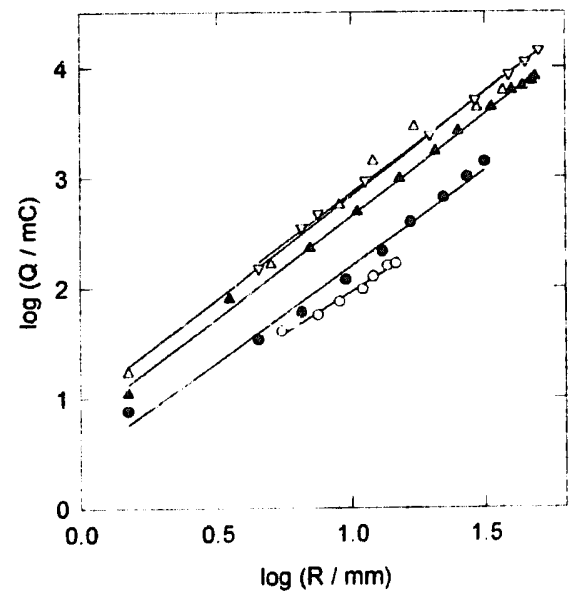

Fig. 10. $\log Q$ vs. $\log \mathrm{R}$ plots at different values of $c:(O) c=0.002 \mathrm{M}$; (O) $c=0.005 \mathrm{M} ;(\Delta) c=0.010 \mathrm{M} ;(\Delta) c=0.014 \mathrm{M} ;(\nabla) c=0.024 \mathrm{M}$. 


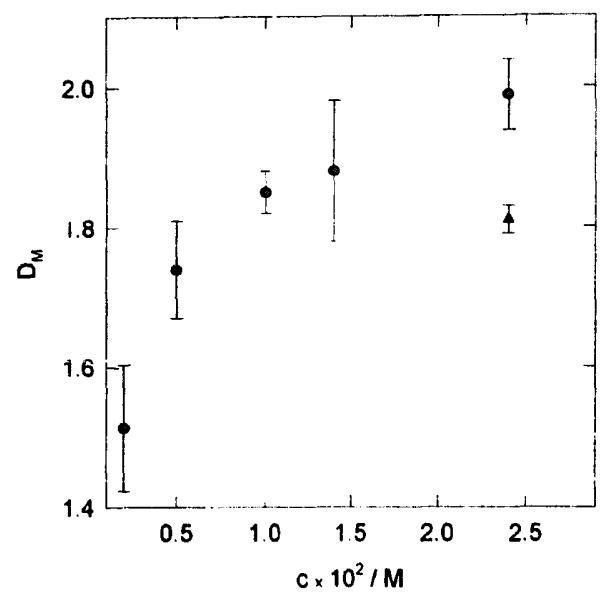

Fig. 11. $D_{M}$ vs. $c$ plot. Data resulting from those experiments run at $\eta_{\mathrm{c}}=0.660 \mathrm{~V}$.

dense to an open radial branching can be seen in the sequential set of photographs resulting from $c=2.4 \times$ $10^{-2}$ and $\eta_{\mathrm{c}}=0.760 \mathrm{~V}$ (Fig. 6(a)-(f)). In any case, the second growth mode transition can be observed when $R$ just exceeds the criticail radius $R_{t}$. The value of $R_{t}$ and the range of ime in which a specific growth mode prevails can be determined from the photographic sequences described above. Furthermore, it appears titat the inner dense portion of electrodeposits remains frozen for $t>t_{\mathrm{t}}$.

\subsubsection{Current transient data under a constant applied potential}

Current transients related to growth patterns obtained for $\eta_{c}=0.660 \mathrm{~V}$ and different $c$ (Fig. 7(a)) exhibit at least two regimes for the rate of current increase, depending on whether $t<t_{\mathrm{t}}$ or $t>t_{\mathrm{t}}$ respectively, $t_{\mathrm{t}}$ being a $c$-dependent transition time related to the second growth mode transition which can be determined for $R=R_{t}$. It is worth noting that the initial decreasing current transient regime which is related to the early stages of growth is not shown in Fig. $7(a)$ as it involves only a few seconds. A detailed discussion of the early stages of growth has been reported elsewhere $[28,29]$.

After the first growth mode transition an almost linear current increase can be observed followed by a smoother rise in current up to $t_{\mathrm{t}}$. Later, for $t>t_{\mathrm{t}}$ an abrupt increase in current can be seen. In the range of $c$ covered by this work, the value of $t_{t}$ increases as $c$ is diminished (Fig. $7(b))$. Considering these data and the corresponding growth patterns, it is possible to derive phenomenological relationships for the dense and open branching growth regimes appearing for $t<t_{\mathrm{t}}$ and $t>t_{\mathrm{t}}$ respectively. Thus, for $t<t_{\mathrm{t}}$ current transients fit the proportionality:

$I \propto t^{m}$

the value of $m$ resulting from the $\log I$ vs. $\log t$ plots (Fig. $7(\mathrm{c})$ ) being in the range $0.6 \leq m \leq 1.0$ (Fig. $7(\mathrm{~d})$ ).

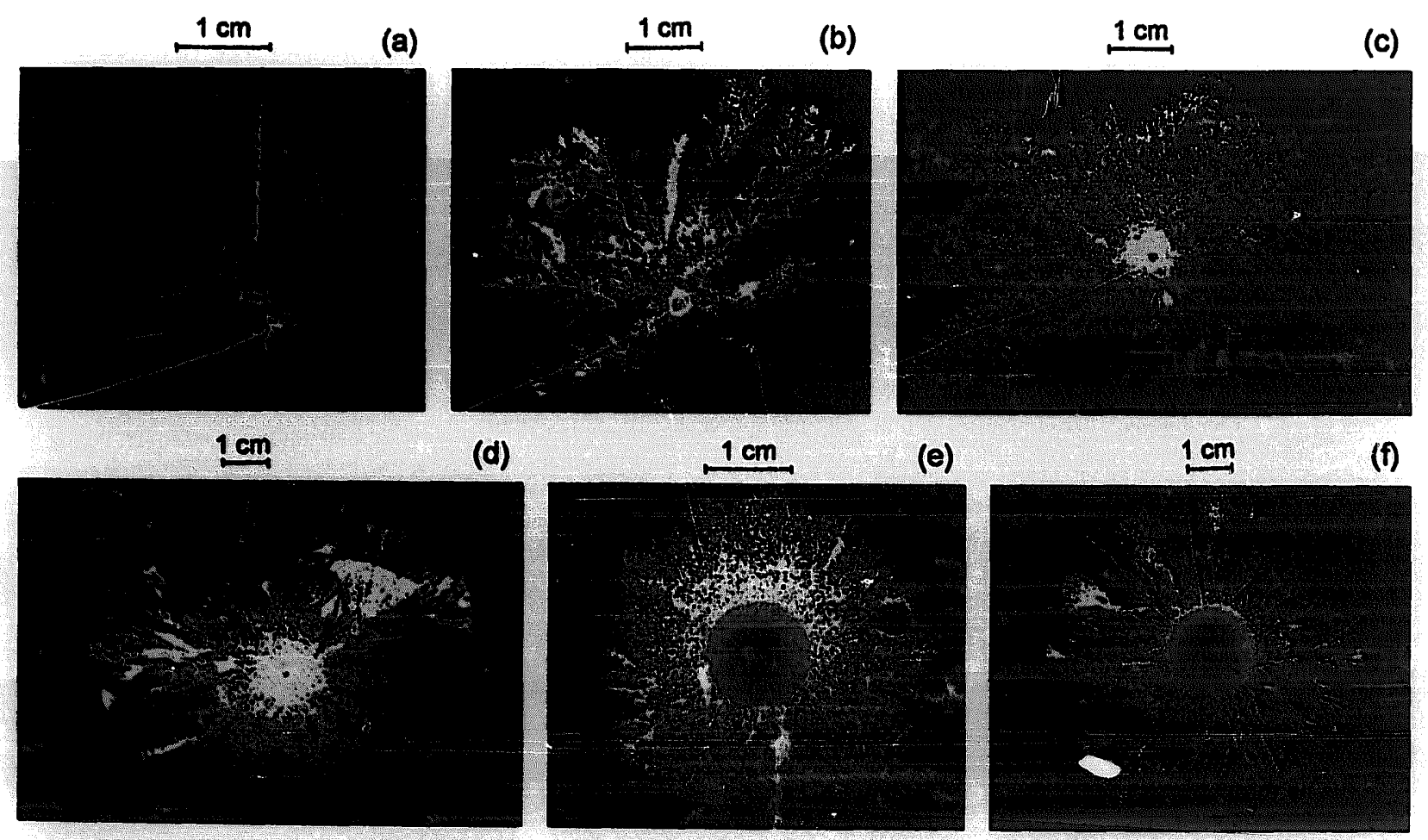

Fig. 12. Photographs of $\mathrm{Ag}$ electrodeposits resulting from aqueous $0.024 \mathrm{M} \mathrm{Ag}_{2} \mathrm{SO}_{4}+0.01 \mathrm{M} \mathrm{H}_{2} \mathrm{SO}_{4}+0.5 \mathrm{M} \mathrm{Na}_{2} \mathrm{SO}_{4}$ electrolyte solution and different values of $\eta_{\mathrm{c}}$ ( (a) $\eta_{\mathrm{c}}=0.118 \mathrm{~V}, Q=2.93 \mathrm{C}$; (b) $\eta_{\mathrm{c}}=0.218 \mathrm{~V}, Q=6.30 \mathrm{C}$; (c) $\eta_{\mathrm{c}}=0.418 \mathrm{~V}, Q=19.2 \mathrm{C}$; (d) $\eta_{\mathrm{c}}=0.518 \mathrm{~V}, Q=16.0 \mathrm{C}$; (e) $\eta_{\mathrm{c}}=0.618 \mathrm{~V}$, $Q=0.359 \mathrm{C}$; (f) $\eta_{\mathrm{c}}=0.760 \mathrm{~V}, Q=9.83 \mathrm{C}$. 
Furthermore, in the same range of time, from the $Q$ vs. $t$ plots (Fig. 8(a)), the following proportionality between $Q$ and $t$ is also established [1-3]:

$Q \propto t^{p}$

where the value of $p$ resulting from the $\log Q$ vs. $\log t$ plot (Fig. 8(b)) changes from $p=1.4$ for $c=10^{-3} \mathrm{M}$ to attain the limiting value $p \cong 2$ for $c \geq 5 \times 10^{-3}$ (Fig. 8(c)).

On the other hand, for growth patterns resulting from $\eta_{c}=0.660 \mathrm{~V}$ and a constant value of $c$, the time-dependence of $\mathbf{R}^{\prime}$ (Fig. 9(a)) fulfils the proportionality

$\mathrm{R}^{\prime} \propto t^{x}$

where $\mathbf{R}^{\prime}=\mathbf{R}-\mathbf{R}_{0}$. Accordingly, from the $\log \mathbf{R}^{\prime}$ vs. $\log t$ plots (Fig. 9(b)) the slope results in $0.9 \leq x \leq 1.1$, irrespective of $c$.

The following correlation between $Q$ and $\mathrm{R}$ at different values of $c$ can also be established [1-3]:

$Q \propto \mathbf{R}^{D_{M}}$
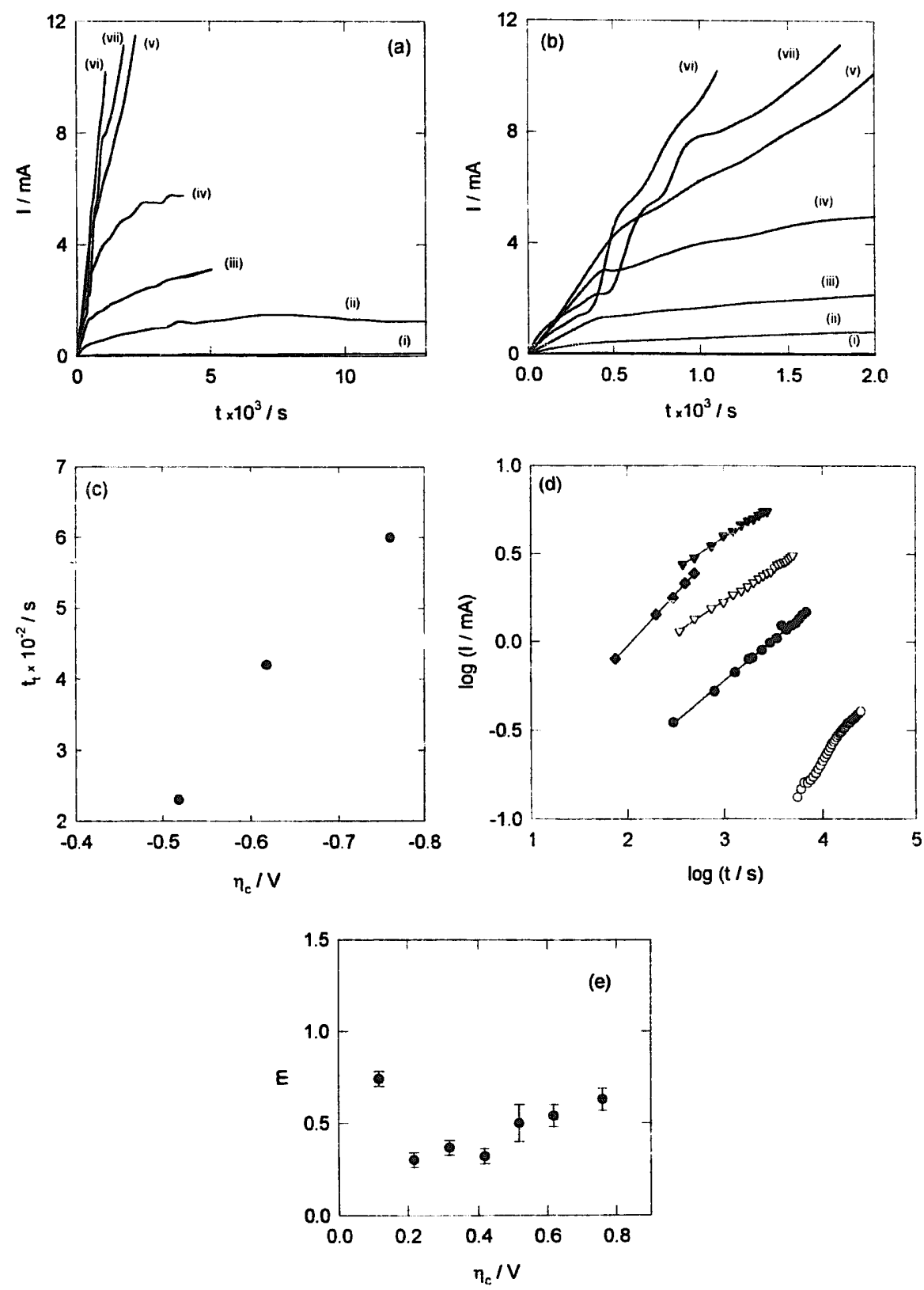

Fig. 13. (a) Current transients for Ag electrodeposits at different values of $\eta_{\mathrm{c}}$. (i) $\eta_{\mathrm{c}}=0.118 \mathrm{~V}$; (ii) $\eta_{\mathrm{c}}=0.218 \mathrm{~V}$, (iii) $\eta_{\mathrm{c}}=0.318 \mathrm{~V}$; (iv) $\eta_{\mathrm{c}}=0.418 \mathrm{~V}$; (v) $\eta_{\mathrm{c}}=0.518 \mathrm{~V}$; (vi) $\eta_{\mathrm{c}}=0.618 \mathrm{~V}$; (vii) $\eta_{\mathrm{c}}=0.760 \mathrm{~V}$. (h) Detail of current transients on a shorter time scale. (c) $t_{\mathrm{c}}$ vs. $\eta_{\mathrm{c}}$ plot. (d) Log $I$ vs. $\log t$ at difterent values of $\eta_{\mathrm{c}}:(O) 0.118 \mathrm{~V}$. (O) $0.218 \mathrm{~V},(\nabla) 0.318 \mathrm{~V},(\nabla) 0.418 \mathrm{~V},(\diamond) 0.760 \mathrm{~V}$. (e) $m$ vs. $\eta_{c}$ plot. Elestrolyte solution: aqueous $0.024 \mathrm{M}$ $\mathrm{Ag}_{2} \mathrm{SO}_{4}+0.01 \mathrm{M} \mathrm{H}_{2} \mathrm{SO}_{4}+0.5 \mathrm{M} \mathrm{Na}_{2} \mathrm{SO}_{4}$. 

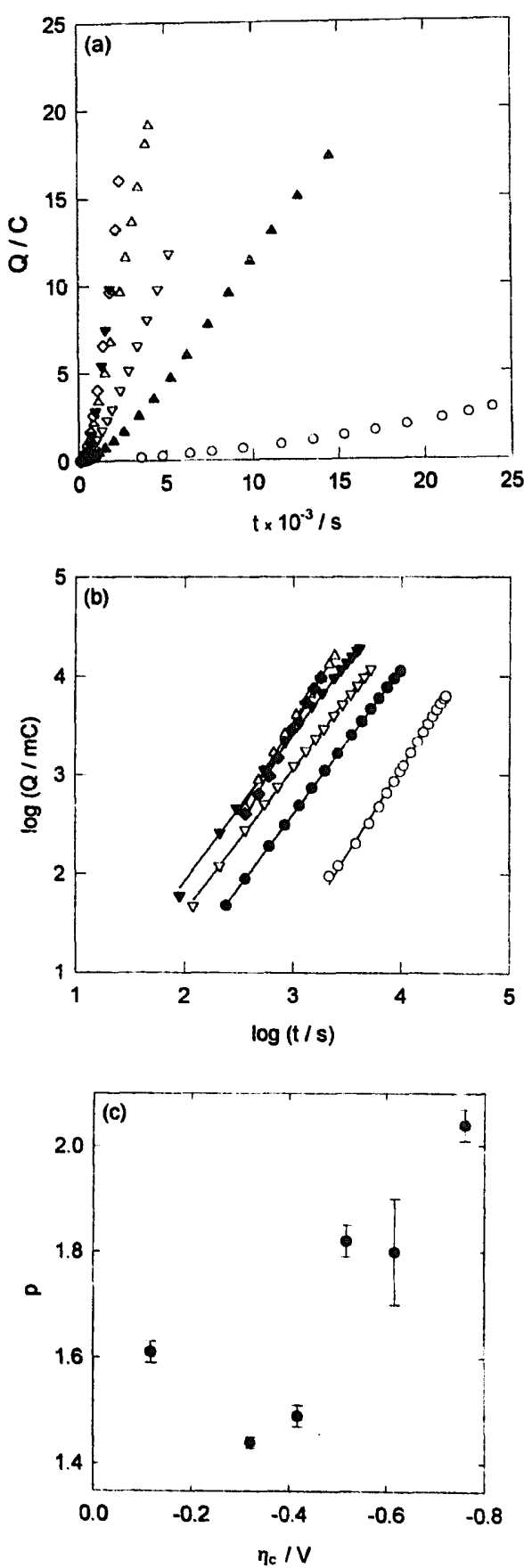

Fig. 14. (a) $Q$ vs. $t$ plots for different values of $\eta_{c}:(O) 0.118 \mathrm{~V},(\Delta)$ $0.218 \mathrm{~V},(\nabla) 0.318 \mathrm{~V},(\Delta) 0.418 \mathrm{~V},(\diamond) 0.518 \mathrm{~V} ;(\diamond) \eta_{c}=0.618 \mathrm{~V} ;(\nabla)$ $0.760 \mathrm{~V}$. (b) $\log Q$ vs. $\log t$ plots, (O) $0.118 \mathrm{~V},(\bigcirc) 0.218 \mathrm{~V},(\nabla)$ $0.318 \mathrm{~V},(\nabla) 0.418 \mathrm{~V},(\Delta) 0.518 \mathrm{~V},(\diamond) 0.760 \mathrm{~V}$. (c) $p$ vs. $\eta_{\mathrm{c}}$ plot.

where $D_{\mathrm{M}}$ is the mass fractal dimension of the growing object. The value of $D_{M}$ can be determined from the $\log Q$ vs. $\log \mathrm{R}$ plot (Fig. 10). The finite value of $Q$ resulting from $R^{\prime} \rightarrow 0$ agrees with the charge value related to the first growth mode transition of the $\mathrm{Ag}$ layer, as has been reported elsewhere $[28,29]$.

Data show that as $c$ is increased from $1 \times 10^{-3}$ to $1 \times 10^{-2}$, the value of $D_{\mathrm{M}}$ increases asymptotically from $D_{\mathrm{M}}=1.5$ to $D_{\mathrm{M}} \rightarrow 2$ (Fig. 11). The former value of $D_{\mathrm{M}}$ is consistent with the formation of an open $2 d$ branching, whereas the latter is that expected for a dense branched morphology [31].

Otherwise, for $c>1 \times 10^{-2}$ the second growth mode transition at $R_{t}$ is accompanied by a change in $D_{M}$ from $D_{\mathrm{M}} \approx 2$ for $\mathrm{R}<\mathrm{R}_{\mathrm{t}}$ to $D_{\mathrm{M}} \approx 1.66$ for $\mathrm{R}>\mathrm{R}_{\mathrm{t}}$. From relationships (2)-(4), it follows that $x=p / D_{\mathrm{M}}$. Thus, as $x \rightarrow 1, p \rightarrow D_{\mathrm{M}}$, a fact which is consistent with data depicted in Fig. 8(c).

\subsection{The influence of $\eta_{c}$ on the growth mode of Ag electrodeposits}

The influence of $\eta_{\mathrm{c}}$ on the growth mode of $\mathrm{Ag}$ electrodeposits, which is reflected in their morphology and the characteristics of current transients, was studied by setting $c=0.024 \mathrm{M}$.

\subsubsection{Transitions in the growth mode}

Ag electrodeposits of a comparable size resulting from $c=0.024$ and different values of $\eta_{c}$ (Fig. 12(a)-(f)) show that the radial symmetry of the growing object increases with $\eta_{c}$. Although the characteristics of these electrodeposits depend considerably on $\eta_{c}$, the two main growth regimes referred to in Section 3.3 can also be distinguished from the corresponding photographs, except in Fig. 12(a) which shows an asymmetric branching approaching a dendritic pattern with a remarkably low branching density. Thus, the size of the inner dense branched region increases almost linearly with $\eta_{c}$ for $\eta_{c} \geq 0.518 \mathrm{~V}$, whereas the morphology of the outer branched region changes substantially with $\eta_{c}$ in the following way. For $\eta_{c}=0.218 \mathrm{~V}$, the branching density increases, but the arm thickness is not uniform (Fig. 12(b)); for $\eta_{i}=0.418 \mathrm{~V}$, there is a more evenly distributed branching (Fig. 12(c)); for $\eta_{c}=0.518 \mathrm{~V}$, branching with a number of rather large spots with a high branching density can be seen (Fig. 12(d)). This description can be extended to those deposits produced for $\eta_{c}=$

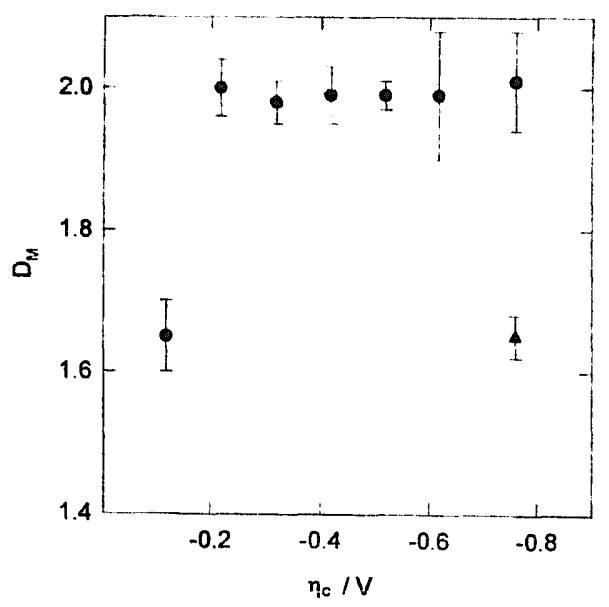

Fig. 15. $D_{\mathrm{M}}$ vs. $\eta_{\mathrm{c}}$ plot. Data resulting from experimental runs in aqueous $0.024 \mathrm{M} \mathrm{Ag}_{2} \mathrm{SO}_{4}+0.01 \mathrm{M} \mathrm{H}_{2} \mathrm{SO}_{4}+0.5 \mathrm{M} \mathrm{Na}_{2} \mathrm{SO}_{4}$ solution. 
$0.618 \mathrm{~V}$ and $\eta_{\mathrm{c}}=0.760 \mathrm{~V}$ (Fig. 12(e), (f)), although in both cases a radial symmetry of the growing object is attained. Therefore, as $\eta_{c}$ is increased, a more complex pattern with several morphologies is accomplished.

From the preceding analysis it appears that the complex patterns of $\mathrm{Ag}$ electrodeposits involve several transitions in the growth mode which depend on both $\eta_{c}$ and $c$. The most remarkable growth mode transition seen on the centimetre scale is the dense to radial open branching transition. Depending on the experimental conditions, this transi-
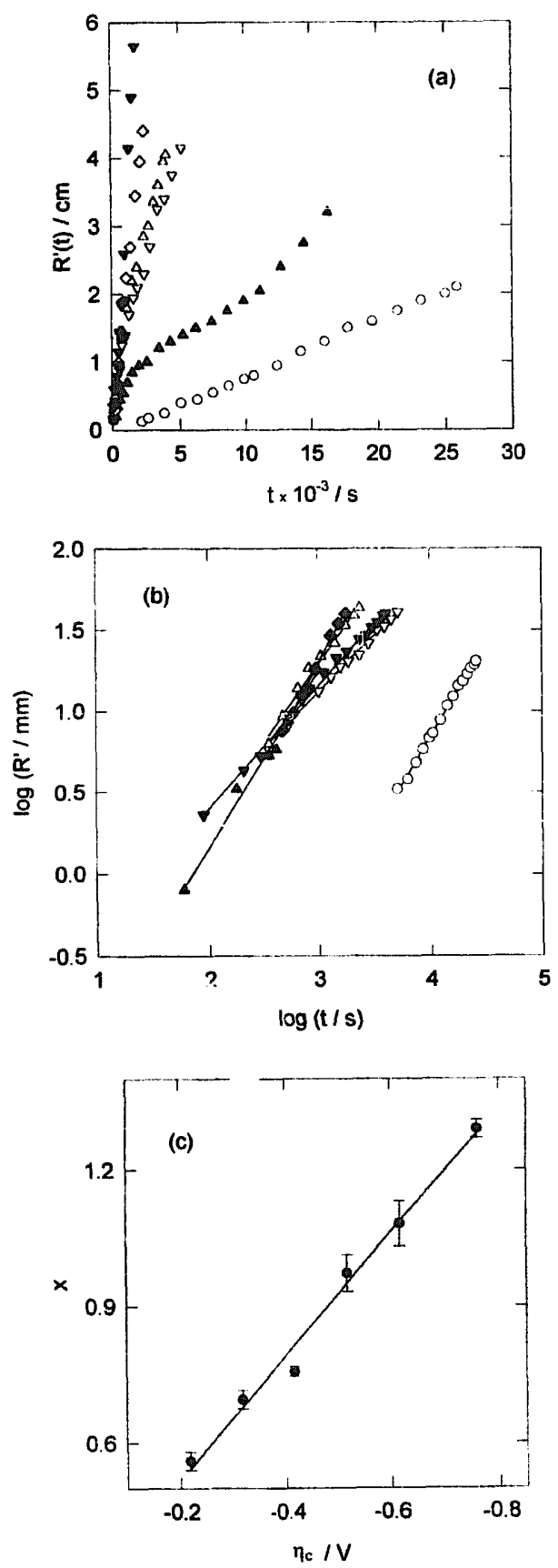

Fig. 16. (a) $\mathrm{R}^{\prime}$ vs. $t$ plots. (O) $\eta_{c}=0.118 \mathrm{~V} ;(\Delta) \eta_{\mathrm{c}}=0.218 \mathrm{~V} ;(\nabla)$ $\eta_{\mathrm{c}}=0.318 \mathrm{~V} ;(\Delta) \eta_{\mathrm{c}}=0.418 \mathrm{~V} ;(\diamond) \eta_{\mathrm{c}}=0.518 \mathrm{~V} ;(\bigcirc) \eta_{\mathrm{c}}=0.618 \mathrm{~V}$; (v) $\eta_{c}=0.760 \mathrm{~V}$. (b) $L \log \mathrm{R}^{\prime}$ vs. $\log t$ plots: (C) $0.118 \mathrm{~V}$, (O) $0.218 \mathrm{~V}$, $(\nabla) 0.318 \mathrm{~V},(\nabla) 0.418 \mathrm{~V},(\Delta) 0.518 \mathrm{~V},(\diamond) 0.760 \mathrm{~V}$. (c) $x$ vs. $\eta_{\mathrm{c}}$ plot. tion is followed by a less remarkable open to dense radial branching transition as the size of the deposit approaches that of the embedding space. This third growth mode transition is presumably associated with the Hecker transition [32,33] and, in principle, it can be related to the potential and current distribution at cells with a radial design as the one used in this work. It was also suggested that this transition is related to a front of hydrogen ions migrating in from the anode [33,34].

\subsubsection{Current transients under a constant potential}

Cathodic current transients for $c=0.024$ run at $\eta_{c} \geq$ $0.218 \mathrm{~V}$ (Fig. 13(a), (b)) exhibit a continuous and slow current increase, whereas those recorded at $\eta_{\mathrm{c}}=0.318 \mathrm{~V}$ show first a small current step extending from $t \rightarrow 0$ to $t \approx 300 \mathrm{~s}$ followed by a continuous current increase. Otherwise, for those runs made in the range $0.518 \mathrm{~V} \leq \eta_{c} \leq$ $0.760 \mathrm{~V}$, a second break in the current transient at longer times can be seen. The time at which this second break appears is related to the change in the growth mode of the electrodeposit already described in Sections 3.2 and 3.3.1.

The transition from the dense to open radial branching pattern is characterized by the transition time $t_{1}$ already referred to that increases with $\eta_{c}$ (Fig. 13íc)).

For $t<t_{t}$, current transients recorded in the range $0.118 \mathrm{~V} \leq \eta_{\mathrm{c}} \leq 0.760 \mathrm{~V}$ plotted as $\log I$ vs. $\log t$ (Fig. 13(d)) also fit the proportionality [1], the corresponding values of $m$ being in the range $0.3 \leq m \leq 1.3$, depending on $\eta_{c}$ (Fig. 13(e)).

On the other hand, the $Q$ vs. $t$ plots (Fig. 14(a)) fit lirear $\log Q$ vs. $\log t$ plots according to expression (2) (Fig. 14(b)), with values of $p$ covering $p \rightarrow 1.5$ for $\eta_{\mathrm{c}} \rightarrow 0$ to $p \rightarrow 2$ for $\eta_{c} \rightarrow 0.760 \mathrm{~V}$ (Fig. 14(c)).

Furthermore, the value of $D_{M}$ derived from the linear $\log Q$ vs. $\log \mathrm{R}$ plots, as predicted by expression (4), increases from $D_{\mathrm{M}}=1.6$ to $D_{\mathrm{M}} \rightarrow 2$ for $\eta_{\mathrm{c}} \geq 0.318 \mathrm{~V}$ (Fig. 15). It is interesting to note that for the growth pattern resulting from $\eta_{c}=0.760 \mathrm{~V}$, the second growth mocis transition is accompanied by a decrease in the value of $D_{\mathrm{M}}$ from $D_{\mathrm{M}} \approx 2$ to $D_{\mathrm{M}} \approx 1.67$, as seen by the single point included in Fig. 15. Otherwise, the $R^{\prime}$ vs. $t$ plots (Fig. 16(a)) fit $\mathrm{R}^{\prime} \propto t^{x}$ relationships (Fig. 16(b)) with values of $x$ increasing linearly with $\eta_{c}$ (Fig. 16(c)).

It should be noted that changes in the pattern morphology with $\eta_{c}$ already described in Section 3.4.1 are accompanied by rhanges in the values of $D_{\mathrm{M}}, m, p$, and $x$.

\section{Discussion}

\subsection{Growth kinetics far from equilibrium}

Experimental data cover two different aspects of $\mathrm{Ag}$ patterns, namely current and charge data which correspond to average information about the global growth process, 
and radial growth data derived from photographs which provide very local information. Taking into account the scale of our experiments and depending on the operating conditions, both global and local data are related to growth modes characterized by either a branched or a dense radial branching followed by either asymmetric or symmetric more or less uniform open branched deposits. Growth patterns reported in this work also show a morphology transition on the centimetre scale between a dense and open radial branching which is reflected in both the density of the aggregates and their symmetry.

Dense radial branching corresponds to symmetric, almost constant density deposits in which tip splitting dominates the interfacial growth. Tip splitting with an apparent density diminishing during growth can also be observed in general in DLA-like patterns. When dense radial aggregates are formed, branches are sufficiently close to one another so that the concentration of the depositing ion in the solution varies practically with the radial direction only. In this case, the aggregate in which tip splitting is produced generates a quasi-homogeneous disc with a resistivity in the order of $10 \Omega \mathrm{cm}$ (see Section 3.1 ).

Conversely, for dendritic patterns an anisotropy, presumably produced either by surface tension or growth kinetics leading to a stable tip behaviour, is observed. The formation of these patterns depends on the experimental conditions including the composition of the electrolyte solution [13,35]. Starting from different growth models, it has been concluded that anisotropy is required to stabilize the growing tip against splitting [36-38].

\subsection{A first approach to the growth mechanism}

Pattern formation resulting from thin layer metal electrodeposition from aqueous solution involves a convective mass transport arising from density gradients that are perpendicular to the gravity field producing a vertical hydrodynamic flow, and an electroconvective transport generated by the electric field at tips causing a horizontal hydrodynamic flow [39]. Due to these contributions a model exclusively based on diffusion as the DLA model cannot describe a number of experimental facts such as certain dense and comb-like patterns, transitions in the global morphology of a growing deposit, and other cooperative effects even at the lowest concentration and lowest applied potential compatible with the faradaic process $[35,39,40]$. However, despite these drawbacks, it has been proposed that the DLA model should capture the essential features of open branching patterns in a number of metal electrodeposition processes, including $\mathrm{Ag}$, at least under a certain range of operating conditions [1-3,41-43].

According to large scale computer simulations for the growth of a branched deposit under the DLA model both in the $2 \mathrm{~d}$ and $3 \mathrm{~d}$ space, the interface motion is determined by random diffusion of depositing particles. This mechanism predicts different parametric relationships among $R$,
$Q, I$ and $t$, depending on whether the radius of the growing object $\mathbf{R}$ is smaller or larger than the diffusion length $l_{\mathrm{d}}$ for depositing particles. Thus, for $\mathrm{R}(t)<l_{\mathrm{d}}$, it follows that $D_{\mathrm{M}}=1.7$ for the $2 \mathrm{~d}$ space, i.e. the object exhibits a fractal mass and obeys the equations

$$
\begin{aligned}
& \mathrm{R}=k_{1} t^{0.6} \\
& Q=k_{2} t \\
& I=k_{3}
\end{aligned}
$$

Conversely, for $\mathrm{R}(t)>l_{\mathrm{d}}, D_{\mathrm{M}}=2$, i.e. a dense branched morphology is produced, and the growth kinetics are dominated by a surface reaction [31]. Then, the following relationships are fulfilled:

$$
\begin{aligned}
& \mathrm{R}=k_{1} t \\
& Q=k_{2} t^{2} \\
& I=k_{3} t
\end{aligned}
$$

Results of this work described in Sections 3.3 and 3.4 indicate that for $\mathrm{Ag}$ electrodeposition in the presence of a supporting electrolyte, the above mentioned limiting situations can only be roughly approached by adjusting either $\eta_{i}$ or $c$. Otherwise, by conditioning one of these variables
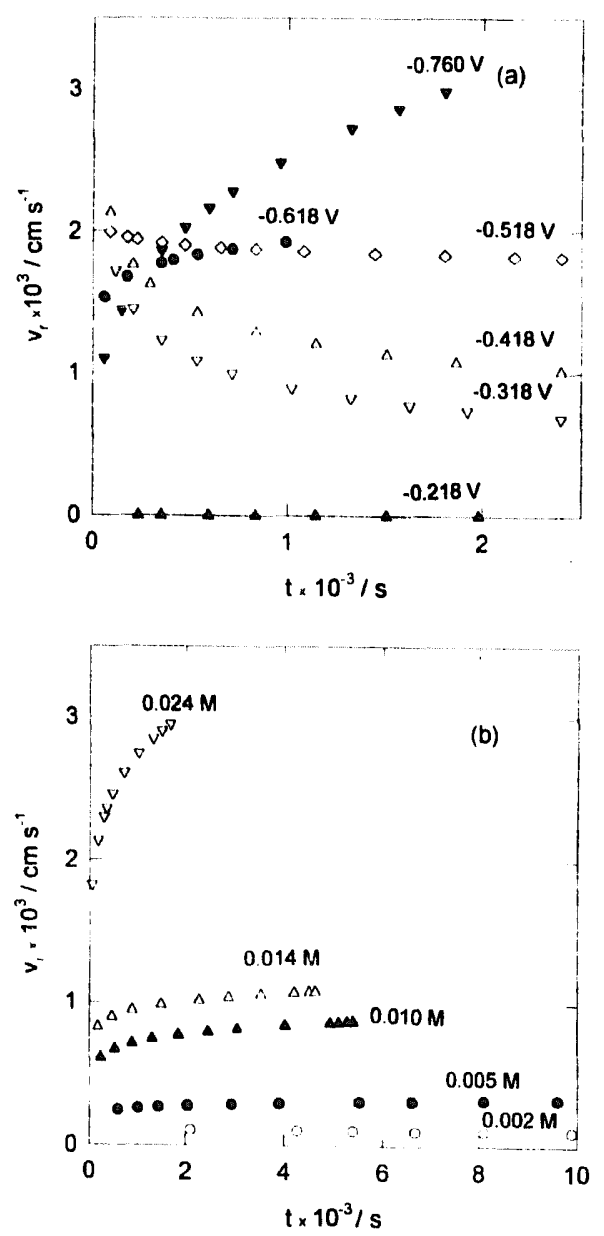

Fig. 17. $v_{r}$ vs. $t$ plots: (a) at constant $c$; (b) at constant $\eta_{c}$. 

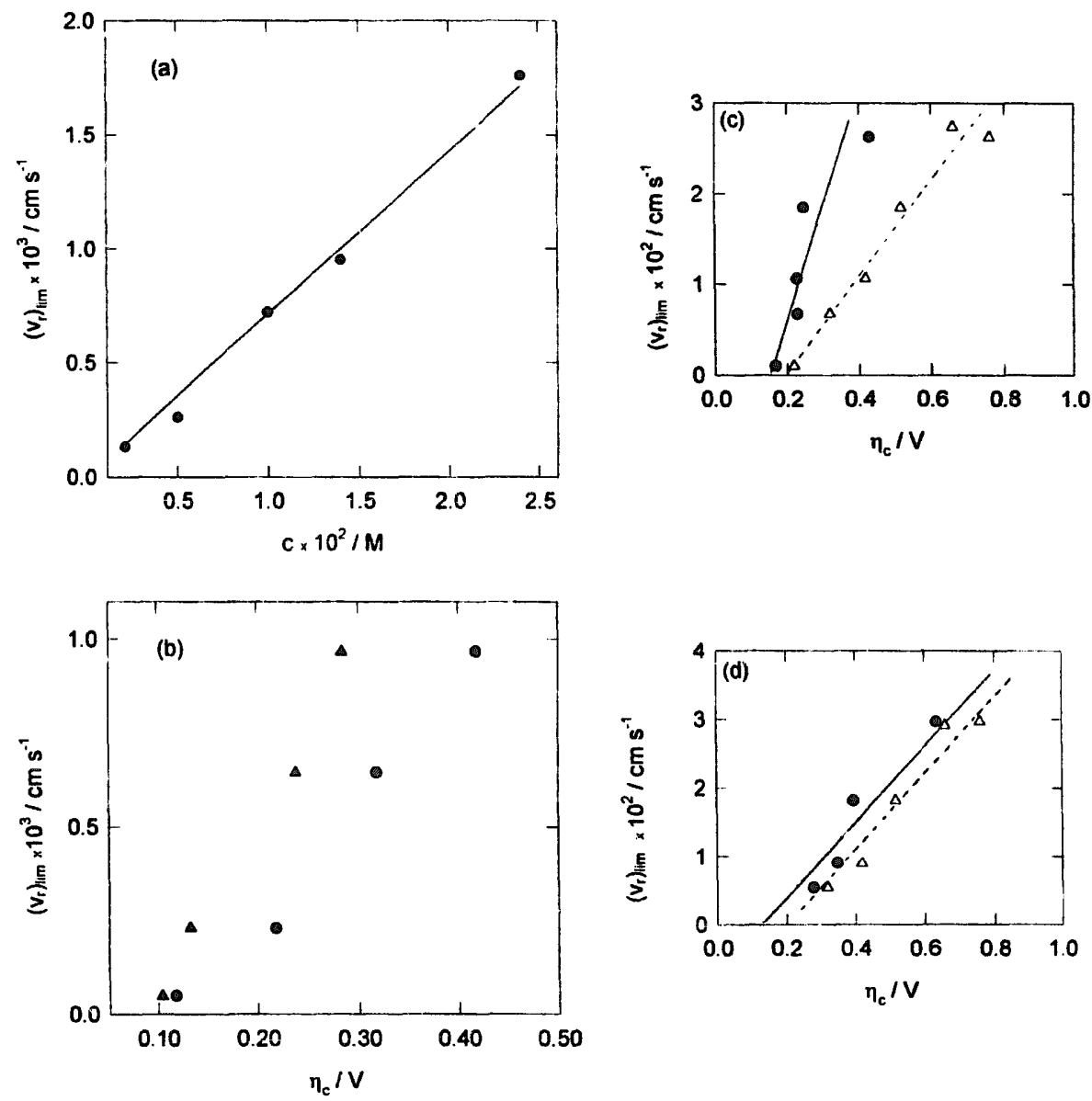

Fig. 18. (a) $\left(v_{\mathrm{r}}\right)_{\text {lim }}$ vs. $c$ plot at $\eta_{c}=0.66 \mathrm{~V}$. (b) $\left(v_{\mathrm{r}}\right)_{\lim }$ vs. $\eta_{\mathrm{c}}$ plot, $(\nabla)$ and $(0)$ correspond to $\eta_{c}$ values with and without ohmic drop correction respectively. (c) $\left(v_{\mathrm{r}}\right)_{\text {tim }}$ vs. $\eta_{c}(\mathrm{R}=3 \mathrm{~cm})$, ( $)$ and $(\Delta)$ correspond to $\eta_{\mathrm{c}}$ values with and without ohmic drop correction respectively. (d) $\left(v_{\mathrm{r}}\right)_{\text {lim }}$ vs. $\eta_{\mathrm{c}}$ $(\mathrm{R}=4 \mathrm{~cm}),(\mathcal{O})$ and $(\Delta)$ correspond to $\eta_{\mathrm{c}}$ values with and without ohmic drop correction respectively.

it is possible to observe a change in the deposit morphology from a DLA-like to a DBM pattern. DBM patterns have been characterized as a fractal surface only at a short length and this has resulted in a smooth envelope for the whole structure [44].

Patterns from $\mathrm{Ag}$ electrodeposits which correspond to DBM patterns fulfil the set of equations predicted by the DLA model only under the limiting condition that the radius of the electrodeposit is greater than the diffusion length of depositing particles. In this case, in agreement with the model, the electrodeposit is characterized by a rather symmetric shape, tip splitting resulting from local instabilities inherent to the diffusion field built around it. Then, an electrodeposit builds up with only a fractal surface and a constant average density.

On the other hand, the DLA model fails to explain the kinetic behaviour of these open branching patterns. In this case, despite the fact that the value of $D_{\mathrm{M}}$ falls within the range $1.5 \leq D_{\mathrm{M}} \leq 1.7$, as expected from either a DLA or a dendritic pattern, the actual exponents are $m=0.6, p=1.4$ and $x=0.9$ instead of $m=0, p=1$ and $x=0.66$ expected for Eqs. (5a), (5b) and (5c). Thence, the parameters derived from experimental data represent an intermediate situation between those two limiting situations described by Eqs. (5a), (5b) and (5c) and Eqs. (6a), (6b) and (6c) respectively $[1-3,41,42]$. This fact suggests that the formation of asymmetric, irregularly shaped deposits involves more complex kinetics in which there is presumably a simultaneous contribution of silver ion diffusion from the

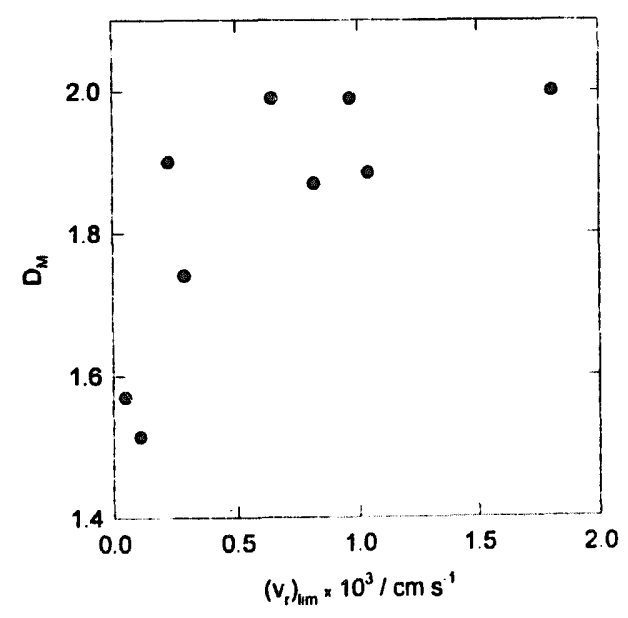

Fig. 19. $D_{\mathrm{M}}$ vs. $\left(v_{\mathrm{r}}\right)_{\mathrm{lim}}$ plot. 
Table ।

Data related to DBM patterns

\begin{tabular}{lllllll}
\hline$c / \mathrm{M}$ & $t / \mathrm{s}$ & $\eta_{\mathrm{c}} / \mathrm{V}$ & $\left(\eta_{\mathrm{c}}-I R\right) / \mathrm{V}$ & $10^{3} I / \mathrm{A}$ & $f_{\mathrm{R}}$ & $10^{3} j / \mathrm{Acm}^{-2}$ \\
\hline 0.024 & 600 & 0.618 & 0.147 & 5.5 & $\sim 1$ & 1.6 \\
0.024 & 600 & 0.760 & 0.385 & 4.3 & $\sim 1$ & 1.3 \\
0.014 & 600 & 0.660 & 0.526 & 1.1 & $\sim 1$ & 0.6 \\
0.010 & 600 & 0.660 & 0.613 & 0.3 & $\sim 1$ & 0.3 \\
0.010 & 2000 & 0.660 & 0.593 & 1.1 & $\sim 60$ & 0.39 \\
0.014 & 2000 & 0.660 & 0.541 & 2.0 & $\sim 60$ & 0.57 \\
0.010 & 2500 & 0.660 & 0.587 & 1.35 & $\sim 60$ & 0.38 \\
0.014 & 2500 & 0.660 & 0.567 & 2.3 & $\sim 60$ & 0.50 \\
\hline
\end{tabular}

solution, and surtace reactions. Accordingly, a non-uniform potential distribution. and a non-uniform diffusion layer thickness should be involved in the process, making the greatest displacements from equilibrium conditions prevail ai those domains of the surface where the electrochemical reaction takes place at the highest rate. Then, the system adopts the fastest growing morphology $[36,45,46]$. Therefore, this type of contribution should also be considerer in the complex processes involved in the electrofor$m \cdot$ ' $n$ of distinct growth patterns in metal electrodepositicn as well as in the transitions between different growth modes.

Finally, it should be borne in mind that from previous results obtained for $3 d$ silver electrodeposit formation, a transition in the growth morphology has been observed due to the interference of convective mass transport [47]. Recently, this type of contribution has received considerable attention and deserves further discussion in terms of the complexity of rate processes and the development of different growth modes in the electrochemical formation of new phases [40].

\subsection{Kinetics of silver ion electrodeposition and growth modes}

Non-uniform surface kinetics imply that different growth modes for $\mathrm{Ag}$ electrodeposition can be expected at different stages of growth and the relative contribution of each growth mode, which is reflected in the value of $D_{\mathrm{M}}$, should depend on both $\eta_{c}$ and $c$. It should be noted that in order to explain a DM pattern at a low $\eta_{\mathrm{c}}$ it is only necessary to introduce anisotropy into the growth process $[29,48]$.

\subsubsection{The radial growth rate}

The dependence of $v_{r}$, the largest front propagation velocity which is determined by the fastest growing arm, on both $\eta_{\mathrm{c}}$ and $c$ becomes particularly relevant to learning about the kinetics and growth modes of $\mathrm{Ag}$ electrodeposits in the presence of a supporting electrolyte.

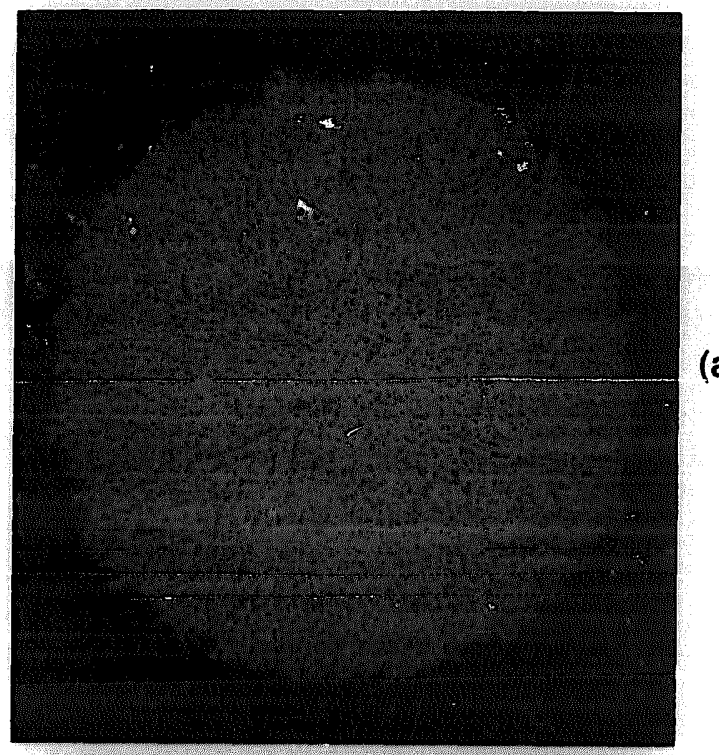

(a)
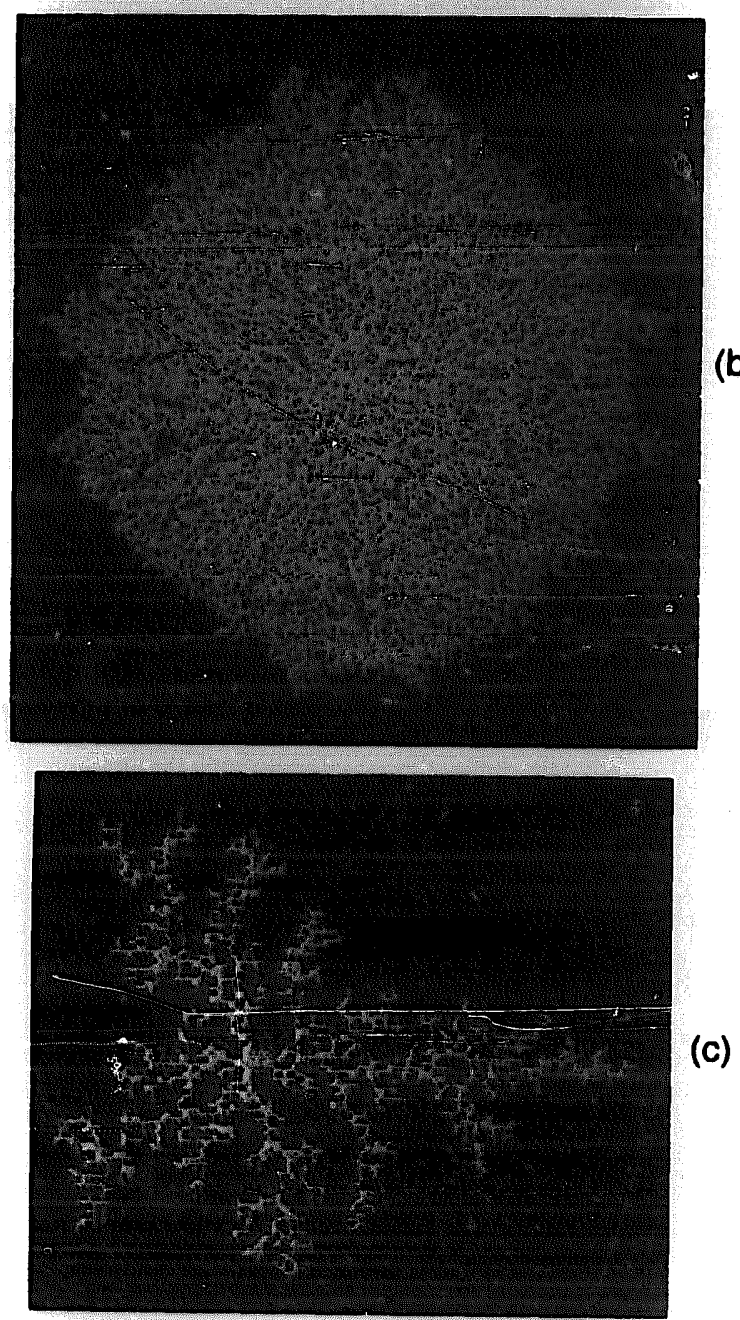

Fig. 20. Monte Carlo snapshots resulting from different sets of kinetic conditions: (a) 10\% diffusion, 100\% sticking probability; (b) $30 \%$ diffusion, $100 \%$ sticking probability; (c) $100 \%$ diffusion, $100 \%$ sticking probability; (d) $0 \%$ diffusion, $100 \%$ sticking probability; (e) $100 \%$ diffusion, $10 \%$ sticking probability: (f) $100 \%$ diffusion, $50 \%$ sticking probability; (g) $100 \%$ diffusion, $70 \%$ sticking probability. 


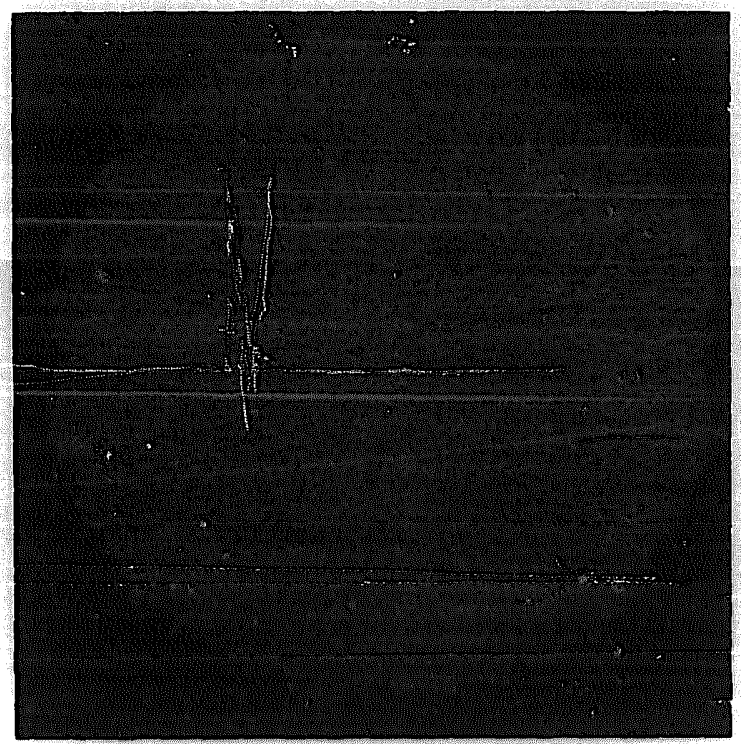

(d)
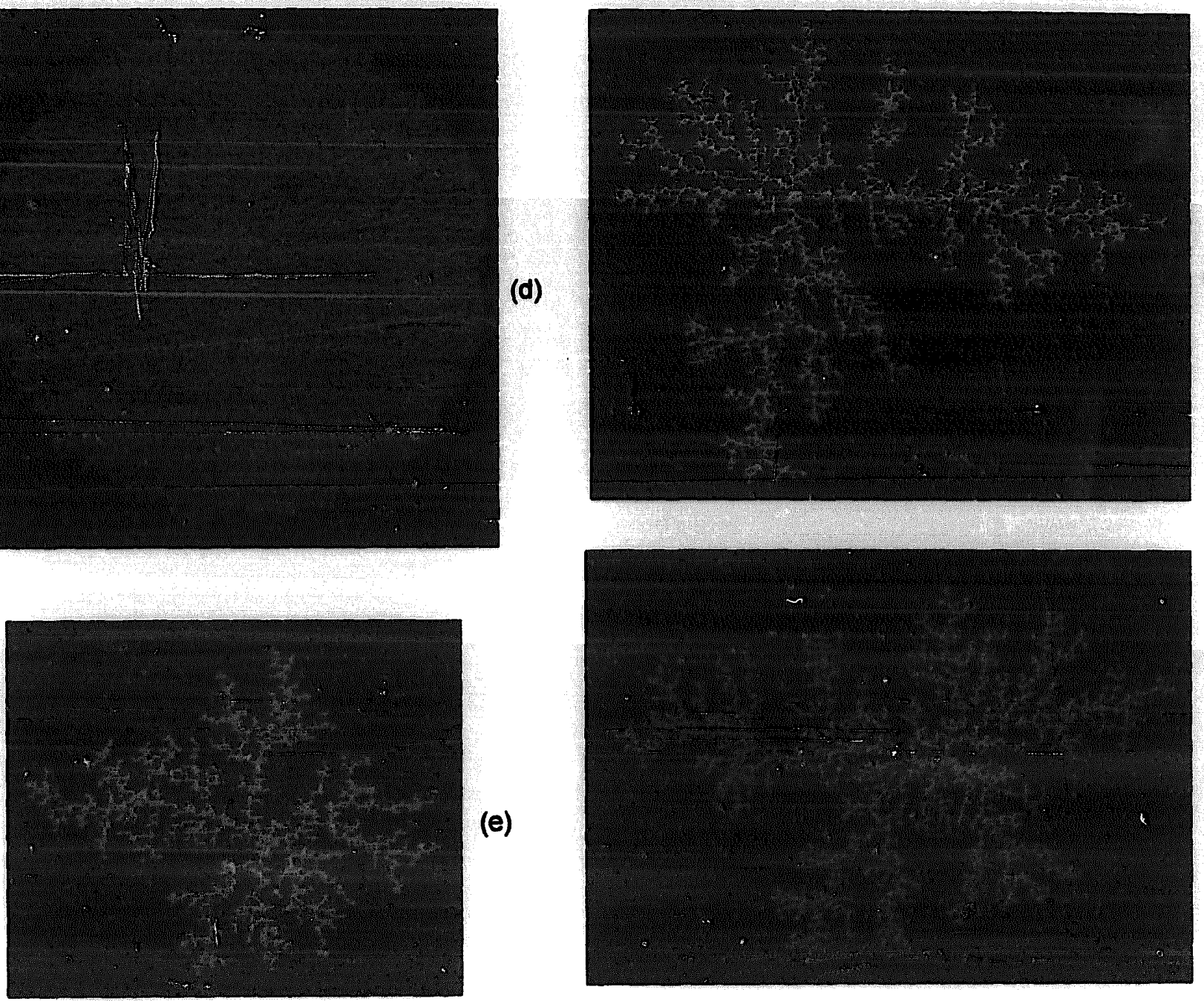

(g)

Considering the definition of $v_{\mathrm{r}}$ and the dependence of $\mathbf{R}^{\prime}$ on $t$, the rate of the local process results in

$v_{\mathrm{r}}=\frac{\mathrm{dR}^{\prime}}{\mathrm{d} t}=a x t^{(x-1)}=k t^{(x-1)}$

where the values of $a$ and $x$ depend $v$. is oth $\eta_{c}$ and $c$. These dependences can be expressed as a single $\eta_{c^{-}}$and $c$-dependent term, $k=k\left(\eta_{\mathrm{c}}, c\right)$. Values of $v_{\mathrm{r}}$ calculated from Eq. (7) are plotted as $v_{\mathrm{r}}$ vs. $t$ at constant $c$ (Fig. $17(\mathrm{a}))$ and $v_{\mathrm{r}}$ vs. $t$ at constant $\eta_{\mathrm{c}}$ (Fig. 17(b)). These plots show that for either constant $\eta_{i}$ or $c$, a limiting value of $v_{\mathrm{r}},\left(v_{\mathrm{r}}\right)_{\lim }$, is attained as $t$ is increased. From these plots for $t \geq 10^{3} \mathrm{~s},\left(v_{\mathrm{r}}\right)_{\mathrm{tim}}$ increases linearly with either $\eta_{\mathrm{c}}$ or $c$ (Fig. 18(a), (b)). A similar result is obtained at a constant R (Fig. 18(c), (d)). These plots also compare the dependences of $\left(v_{\mathrm{r}}\right)_{\text {lim }}$ on both $\eta_{c}$ and $\eta_{c}$ after ohmic drop correction, for different radii of the deposit. Obviously, the ohmic drop correction has a remarkable effect, although it does not change, at least within experimental error, the type of functionality. In addition, these plots show that there is a threshold potential $\eta_{0}$ which is associated with the triggering of branching growth. For our system it results in $\eta_{0}=0.10 \pm 0.03 \mathrm{~V}$. Therefore, for $t \geq 2 \times 10^{3} \mathrm{~s}$, the growth of the largest arms can be described by the simple equation

$v_{\mathrm{r}}=k(t) c\left(\eta_{\mathrm{c}}-\eta_{0}\right)$

By adjusting values of $v_{r}, \eta_{c}$ and $c$ the value of $k(t)$ can be obtained. For $t \geq 2 \times 10^{3} \mathrm{~s}$, the value $k(t)=0.20 \pm$ $0.03 \mathrm{~cm} \mathrm{~s}^{-1} \mathrm{M}^{-1} \mathrm{~V}^{-1}$ is obtained. The value of $\left(v_{\mathrm{r}}\right)_{\mathrm{lim}}$ of those runs in which $\eta_{c}$ and $c$ were simultaneously changed can also be estimated.

For $t \geq 2 \times 10^{3} \mathrm{~s}$, the value of $\eta_{c}$ after ohmic drop correction plays a main role in driving tip growth. A similar conclusion based on the applied potential has been previously reported for $\mathrm{Cu}$ electrodeposition in a $2 \mathrm{~d}-\mathrm{elec}-$ trochemical cell in the presence of a supporting electrolyte $[17,46]$.

The preceding analysis supports the idea that there is a substantial contribution of both an electric and a diffusional field around the growing tip which produces a biased random walk of diffusing ions on the solution side. 
This effect maximizes on the solution side adjacent to the metal electrode where the electroneutrality condition in the system is no longer maintained. Hence, from the standpoint of electrochemical kinetics, the linear $v_{r}$ vs. $\eta_{c}$ plot indicates that in this case the supporting electrolyte does not suppress the influence of the elcctric field on $\mathrm{Ag}$ growing tips. This conclusion applies to either DLA, DM or DBM Ag electrodeposit morphologies.

On the other hand, the $\left(v_{\mathrm{r}}\right)_{\text {lim }}$ vs. $D_{\mathrm{M}}$ plot (Fig. 19) clearly indicates that small changes in $v_{\mathrm{r}}$ are related to a sharp change from the open branching patterns $\left(D_{\mathrm{M}}=1.5\right)$ to the dense radial morphology $\left(D_{\mathrm{M}}=2\right)$

It should be remembered that computer simulations for the DLA model show transitions from those patterus with $D_{M}=1.67$ to thin ribbon-like patterns with $D_{M}=1.0$ which can be produced when the front propagation velocity follows a power dependence on the applied electric field, i.e. the so-called $\eta$-model $[49,50]$.

\subsubsection{Dense branching morphologies}

For the DBM the dependence of $j$, the current density referred to the area of the expanding front, on $\eta_{c}$ and $c$ gives information about the growth of the overall obiect. The value of $j$ was obtained from the ratio $I / S$, where $I$ is the instantaneous current and $S$, the electrode area, is $S=2 \pi h \mathrm{R} f_{\mathrm{R}}$, where $h$ is the thickness of the quasi-2d cell, and $f_{\mathrm{R}}$ is a roughness factor which was estimated from the $\mathrm{Pb}$ and $\mathrm{Cd}$ underpotential deposition voltammograms for 3d dendritic $\mathrm{Ag}$ deposits already reported [28]. Data assembled in Table 1 show that $j$ increases linearly with $c$ and becomes practically independent of $\eta_{\text {a }}$ after ohmic drop correction as should be expected for a process which is under mass transport rate control. These tesults are then consistent with DBM patterns resulting from is by theoretical models [1-3].

Finally, under certain circumstances the system changes from an open fractal structure to a DBM when the aggregate exceeds a certain size. This transition, which resembles the Hecker effect $[32,33]$, has been related is tite distance between filaments of the aggregate and the branching mechanism itself [27]. A spatio-temporal morphological transition of this type has been observed for $\mathrm{Zn}$ electrodeposition using a thin gap elecirochemical cell [40].

\subsection{Monte Carlo simulations}

Monte Carlo simulations have become a very convenient tool to provide a physical picture of growth processes $[1-3,7]$. By an adequate choice of an applied potential-dependent sticking probability and concentration, different growth patterns have been obtained [31].

Our experimental results show that both the applied potential and the concentration dependence of growth pattems are qualitatively reflected in the values of $p, x$ and $D_{\mathrm{M}}$. This situation encouraged us to derive the values of these parameters from Monte Carlo simulation growth patterns.

For this purpose, the influence of the diffusion and electric field on metal electrodeposition was studied through 2d Monte Carlo simulations based on the classical aggregation model of Witten and Sander [41] with the addition of a biased random walk contribution [51]. In this way we attempted an estimation of the influence of the biased walk on $x, p$ and $D_{\mathrm{M}}$. The simulation was carried out on a square lattice $640 \times 480$ points which has an initial seed at the centre. Briefly, particles are released one by one at a constant distance larger than the maximusti radius of the deposit and are permitted to follow a random walk. When a particle sticks at any point of the interface, it becomes irreversibly a part of thic aggregate. Otherwise, when the position of the particle exceeds a certain preset maximum distance, the particle is cleared out and another one is released at random. The length scale of the random walk is always much larger than the size of the growing object.

The influence of the electric field was simulated by a biased random walk introducing the parameter $P_{\mathrm{n}}$. When the radial direction is the only permitted displacement $\left(P_{\mathrm{n}}=1: 0 \%\right.$ diffusion) the particle is allowed to move only if its new position is closer to the growing interface. In this case a dense deposit is formed with $D_{M}=1.95$. Conversely, in the absence of an external electric field growth operates under $100 \%$ diffusion $\left(P_{\mathrm{n}}=0\right)$. Under this condition a typical DLA-like deposit is obtained with a fractal dimension $D_{M}=1.7$.

The snapshots resulting from aggregates grown for
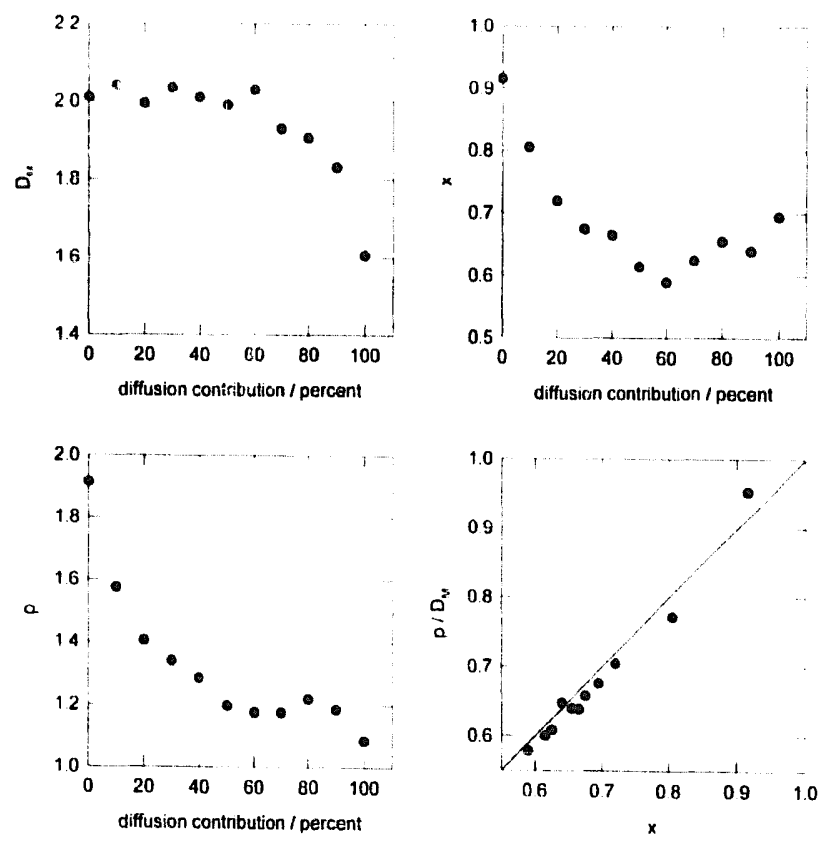

Fig. 21. Parametric relationships derived from the Monte Carlo snapshots. (a) $D_{\mathrm{M}}$ vs. percentage of diffusion contribution plot. (b) $x$ vs. percentage of diffusion contribution plot. (c) $p$ vs. percentage of diffusion contribution plot. (d) $p / D_{\mathrm{M}}$ vs. $x$ plot. The straight line slope is equal to 1 . 
$100 \%$ diffusion and $0 \%$ diffusion resemble closely those found for low $\eta_{c}$ and high $\eta_{c}$ values respectively, before the transition to the open pattern (Fig. 20). This means that on average the contribution of diffusion decreases as $\eta_{\mathrm{c}}$ is increased. The $x, p, D_{\mathrm{M}}$ and $p / D_{\mathrm{M}}$ vs. percentage diffusion plots follow the same trend observed for the experimental $x, p, D_{\mathrm{M}}$ and $p / D_{\mathrm{M}}$ vs. $\eta_{c}$ plots (Fig. 21). The decrease in the random walk relative contribution results in the progressive increase in $x, p$ and $D_{\mathrm{M}}$ approaching the values experimentally found for high $\eta_{c}$. This agreement suggests that the morphology of the object is to a large extent determined by the combined effect of a random and biased walk acting on the depositing particles [27] even in the presence of a supporting electrolyte. This is not surprising as in electrochemical reactions the driving force always involves the electrochemical potential gradient built up on the solution side, particularly in those regions where the electroneutrality condition is no longer obeyed.

\section{Conclusions}

Growing patterns resulting from silver electrodeposition, under a constant applied potential in a quasi-2d electrochemical cell in the presence of a supporting electrolyte, depend on the concentration of silver ions in the solution and on the applied electric potential.

On the centimetre scale, two main types of growing patterns can be distinguished corresponding to a dense branching and an open branching morphology, the latter also including dendritic patterns.

The development of DBM is favoured as either the silver ion concentration or the applied negative potential is increased. However, a transition from DBM to open branching can also be observed. This transition operates after the deposit has reached a critical size which depends on the applied potential and silver ion concentration.

The DBM is related to an almost spherically symmetric electrodeposit which is characterized by tip splitting and a fractal surface. Conversely, open branching morphologies are asymmetric and exhibit mass and surface fractality. These morphologies show a steady growth of stable tips.

The behaviour of DBM fulfils the predictions of the DLA model only in the limiting case in which the radius of the deposit exceeds the diffusion length of depositing particles. Otherwise, the behaviour of open branching morphologies does not follow the predictions of the DLA model currently used for new phase growth models far from equilibrium.

The fact that the silver elcctrodeposition reaction rate involves the electrochemical potential gradient as driving force even in the presence of a supporting electrolyte can explain why models proposed on the basis of a single potential field and particle motion type are limited in accounting for the experimental data.
A Monte Carlo simulation provides a way to understand the complexity of the problem by using a composed probability of particle attachment in a growth process such as that involved in this work.

\section{Acknowledgements}

The authors are indebted to the Consejo Nacional de Investigaciones Científicas y Técnicas (CONICET) of Argentina and the Comisión de Investigaciones Científicas de la Pcia. de Bs. As. (CIC) for financial support of this work.

\section{References}

[1] P. Meakin, in D. Avnir (Ed.). The Fractal Approach to Heterogeneous Chemistry, Surfaces, Colloids and Polymers, Wiley, London, 1990, Chapter 3.1.2, p. 131.

[2] C. Godrèche (Ed.), Solids far from Equilibrium, Cambridge University Press. Cambridge, 1992.

[3] A.L. Barabasi, H.E. Stanley, Fractal Concepts in Surface Growth, Cambridge University Press, Cambridge, 1995.

[4] J. Stranski, L. Krastanov, Akad. Wiss. Math. Nat. KIIlb (1938) 797.

[5] F. Franck, J. van der Merwe, Proc. Roy. Soc. London, Ser. A 198 (1949) 205.

[6] M. Volmer, A. Weber, Z. Phys. Chem. 119 (1926) 277.

[7] P. Meakin, Hetero. Chem. Rev. I (1994) 99.

[8] T. Halpin-Healy, Y-Ch. Zhang, Phys. Rep. 254 (1995) 215.

[9] M. Matsushita, Y. Hayakawa, H. Honjo, Y. Sawada. Phys. Rev. Lett. 53 (1984) 286.

[10] M. Matsushita, Y. Hayakawa, Y. Sawada, Phys. Rev. A 32 (1985) 3814 ,

[11] D.G. Grier. E. Ben-Jacob, R. Clarke, L.M. Sanders, Phys. Rev. Lett. 56 (1986) 1264.

[12] Y. Sawada. A. Dougherty, J.P. Gollub, Phys. Rev. Lelt. 56 (1986) 1260.

[13] D.G. Grier. D.A. Kessler. L.M. Sander, Phys. Rev. Lell. 59 (1987) 2315.

[14] F. Argoul, A. Arneodo, G. Grasseau, H.L. Swinney, Phys. Rev. A 61 (1988) 2558.

[15] F. Argoul, A. Arneodo, J. Elezgaray, G. Grasseau. R. Murenzi, Phys. Rev. A 41 (1990) 5537.

[16] M.A. Guzman, R.D. Feimuth, P.U. Prendse, M.C. Veindt, L. Lam. in L. Lam, H.C. Morris (Eds.), Nonlinear Structures in Physical Systems, Springer, New York, 1990.

[17] P.P. Trigueros, J. Claret, F. Mas, F. Sagués, J. Electroanal. Chem. $312(1991) 219$.

[18] A. Kuhn. F. Argoul, J. Electroanal. Chem. 397 (1995) 93.

[19] M.E. Vela, J.L. Zubimendi, P. Ocón, P. Herrasti, R.C. Salvarezza, L. Vázquez, A.J. Arvia, Electrochim. Acta 41 (1996) 1891.

[20] M.E. Vela, G. Andreasen, R.C. Salvarezza. A.J. Arvia, J. Chem. Soc.. Faraday Trans., 92 (1996) 4093.

[21] J.H. Kaufman. A.I. Nozzel, O. Melroy, A. Kapitulnik, Pnys. Rev. B 35 (1987) 1881.

[22] J.R. Meirose, D.B. Hibbert. Phys. Rev. A 40 (1989) 1727.

[23] V. Fleury, M. Rosso. J.-N. Chazalviel, B. Sapoval, Phys. Rev. A 44 (1991) 6693.

[24] R.M. Brady,, R.C. Ball, Nature 309 (1984) 225.

[25] Y. Deng. M. Pritzker. J. Electroanal. Chem. 336 (1992) 25.

[26] R. Duo, M.J. Peña, R. Celdrán, J. Electroanal. Chem. $4(14$ (1996) 99.

[27] J.-N. Chazaviel, Phys. Rev. A 42 (1990) 7355.

[28] A. Hernández Creus, P. Carro, S. González, R.C. Salvarezza, A.J. Arvia, J. Electrochem. Soc. 139 (1992) 1064. 
[29] H. Martín, P. Carro, A. Hernándezz Creus, S. González, R.C. Salvarezza, A.J. Arvia, Langmuir, 13 (1997) 100.

[30] B. Mandelbrot, in D. Avnir (Ed.). The Fractal Approach to Heterogeneous Chemistry, Surfaces, Colloids and Polymers, Wiley, London, 1990, Chapter 1.3, p. 45.

[31] P. Meakin, J.M. Deutch, J. Chem. Phys. 80 (1984) 2115.

[32] N. Hecker, D.G. Grier, L.M. Sanders, in R.B. Laibowitz, B.B. Mandelbrot, D.E. Passoja (Eds.), Fractal Aspects of Materials, Materials Research Society, University Park, PA, 1985.

[33] V. Fleury, M. Rosso, J.-N. Chazaviel, ? iiy. . Rev. A 43 (1991) 6908.

[34] J.R. Melrose, D.B. Hibbert, R.C. Bali, Phys. Rev. Lett. 65 (1990) 3009.

[35] P.L. Schilardi, S.L. Marchiano, R.C. Salvarezza, A.J. Arvia, in preparation.

[36] E. Ben-Jacob, P. Garik, Nature 343 (1990) 523.

[37] D. Grier, E. Ben-Jacob, R. Clarke, L.M. Sander, Phys. Rev. Lett. 56 (1986) 1264

[38] J.S. Langer, Rev. Mod. Phys. 52 (1980) 1.

[39] J.M. Huth, H.L. Swinney, W.D. McCormick, A. Kuhn, F. Argoul, Phys. Rev. E 51 (1995) 3444.
[40] A. Kuhn, F. Argoul, Phys. Rev. E 49 (1994) 4298.

[41] T.A. Witten Jr., L.M. Sander, Phys. Rev. Lett. 47 (1981) 1400.

[42] T.A. Witten Jr., L.M. Sander, Phys. Rev. B 27 (1983) 5686.

[43] W.Y. Tam, J.J. Chae, Phys. Rev. A 43 (1991) 4523.

[44] M. Wang, N. Ming, Phys. Rev. Lett. 71 (1993) 113.

[45] D. Barkey, P. Garik, E. Ben-Jacob, B. Miller, B. Orr, J. Electruchem. Soc. 139 (1992) 1044.

[46] D. Barkey, F. Oberholtzer, Q. Wu, Phys. Rev. Lett. 75 (1995) 2980.

[47] P. Carro, S.L. Marchiano, A. Hernández Creus, S. González, R.C. Salvarezza, A.J. Arvia, Phys. Rev. E 48 (1993) R2374.

[48] Y. Sawada, A. Dougherty, J.P. Gollub, Phys. Rev. Lett. 56 (1986) 1260.

[49] L. Niemeyer, L. Pietronero, H.J. Wiesmann, Phys. Rev. Lett. 52 (1984) 1033.

[50] A. Sanchez, F. Guinea, L.M. Sander, V. Hakim, E. Louis, Phys. Rev. E 48 (1993) 1296.

[51] A. Hernández Creus, P. Carro, S. González, S. Marchiano, R C. Salvarezza, A.J. Arvia, in M.M. Novak (Ed.), Fractals in the Natural and Applied Sciences, vol. A4I, North Holland, Amsterdam, 1994, p. 191. 